\title{
STABILITY OF MULTI-DIMENSIONAL VISCOUS SHOCKS FOR SYMMETRIC SYSTEMS WITH VARIABLE MULTIPLICITIES
}

\author{
TOAN NGUYEN
}

\begin{abstract}
We establish long-time stability of multi-dimensional viscous shocks of a general class of symmetric hyperbolic-parabolic systems with variable multiplicities, notably including the equations of compressible magnetohydrodynamics (MHD) in dimensions $d \geq 2$. This extends the existing result established by K. Zumbrun for systems with characteristics of constant multiplicity to the ones with variable multiplicity, yielding the first such a stability result for (fast) MHD shocks. At the same time, we are able to drop a technical assumption on structure of the so-called glancing set that was necessarily used in previous analyses. The key idea to the improvements is to introduce a new simple argument for obtaining a $L^{1} \rightarrow L^{p}$ resolvent bound in low-frequency regimes by employing the recent construction of degenerate Kreiss' symmetrizers by O. Guès, G. Métivier, M. Williams, and K. Zumbrun. Thus, at the low-frequency resolvent bound level, our analysis gives an alternative to the earlier pointwise Green's function approach of K. Zumbrun. High-frequency solution operator bounds have been previously established entirely by nonlinear energy estimates.
\end{abstract}

\section{Contents}

1. Introduction

1.1. Equations and assumptions

1.2. Shock profiles

1.3. The uniform Evans stability condition

1.4. The GMWZ result

1.5. Main results

1.6. Discussion and open problems

2. Linearized estimates

2.1. High-frequency estimate

2.2. $L^{2}$ stability estimate for low frequencies

2.3. $\quad L^{1} \rightarrow L^{p}$ estimates

2.4. Estimates on the solution operator

2.5. Proof of linearized stability

3. Nonlinear stability

Date: Revised date: November 6, 2018.

2000 Mathematics Subject Classification. Primary 35L60; Secondary 35B35, 35B40.

I would like to thank Professor Kevin Zumbrun for suggesting the problem and his many great advices, support, and helpful discussions. I also thank the referees for their helpful comments that greatly improved the exposition. This work was supported in part by the National Science Foundation award number DMS0300487. 
4. Two-dimensional case or cases with (H5)

Appendix A. Evans function for the doubled boundary problem 22

\begin{tabular}{ll|} 
Appendix B. Auxiliary problem & 26 \\
\hline
\end{tabular}

Appendix C. Independence of the pointwise Green bounds $\quad 29$

References

\section{INTRODUCTION}

We consider a general system of viscous conservation laws $(d \geq 2)$

$$
\tilde{U}_{t}+\sum_{j} F^{j}(\tilde{U})_{x_{j}}=\sum_{j k}\left(B^{j k}(\tilde{U}) \tilde{U}_{x_{k}}\right)_{x_{j}}, \quad x \in \mathbb{R}^{d}, \quad t>0,
$$

$\tilde{U}, F^{j} \in \mathbb{R}^{n}, B^{j k} \in \mathbb{R}^{n \times n}, n \geq 2$, with initial data $\tilde{U}(x, 0)=\tilde{U}_{0}(x)$, and a planar viscous shock, connecting the endstates $U_{ \pm}$:

$$
\tilde{U}=\bar{U}\left(x_{1}\right), \quad \lim _{x_{1} \rightarrow \pm \infty} \bar{U}\left(x_{1}\right)=U_{ \pm} .
$$

We study the long-time linearized and nonlinear stability of the viscous shock $\bar{U}$ under multi-dimensional perturbations of initial data. The problem has been carefully and successfully investigated by K. Zumbrun and his collaborators in [Z2, Z3, Z4, GMWZ1]. There, due to technical arguments of the analysis, the authors put assumptions on the multiplicity of hyperbolic characteristic roots and structure of the so-called glancing set (see (H4)-(H5) below). The latter assumption (which is automatically satisfied in dimensions $d=1,2$ and in any dimension for rotationally invariant problems) assures the glancing set to be confined to a finite union of smooth curves on which the branching eigenvalue has constant multiplicity. This is precisely to reduce the complexity of multi-variable matrix perturbation problem when dealing with glancing blocks to a simplified form of a twovariable perturbation problem. Whereas, the constant multiplicity assumption excludes an important physical application, namely, the equations of magnetohydrodynamics (MHD) in dimensions $d \geq 2$. In the current paper, we are able to relax the assumption of constant multiplicities to variable multiplicities, allowing (fast) MHD shocks to be treated and thus yielding for the first time the long-time multi-dimensional stability for these shocks. In addition, we are also able to drop the assumption on structure of the glancing set at a price of having $t^{1 / 4}$ slower in decay rates in dimensions $d \geq 3$.

Our main improvements rely on recent remarkable and technical works of O. Guès, G. Métivier, M. Williams, and K. Zumbrun GMWZ5, GMWZ6] where the authors have obtained the $L^{2}$ stability estimates and small viscosity stability for the symmetric systems with variable multiplicities via their construction of Kreiss' symmetrizers. The idea is to employ these available estimates to establish the long-time stability, or more precisely, to derive a resolvent bound in low-frequency regimes. This will be the main contribution of our present paper. High-frequency estimates are already established by K. Zumbrun via elegant nonlinear energy estimates for a very general class of symmetrizable systems, including our class under consideration. 
We would like to mention that the idea of using $L^{2}$ stability estimates via the construction of degenerate Kreiss' symmetrizers to attack the long-time stability problem has been investigated in GMWZ1]. There the authors obtain the result under (H4)-(H5) assumptions (and treat the strictly parabolic systems). In our analysis, we avoid these technical assumptions, by introducing a rather simpler argument for $L^{1} \rightarrow L^{p}$ resolvent bounds in low-frequency regimes, which turns out to be the key to the improvements. The analysis works precisely for the case of dimensions $d \geq 3$. In dimension $d=2$ (the condition (H5) is now always satisfied), the analysis of GMWZ1 indeed works even for the MHD shocks as we are considering here by combining their later work in GMWZ6] (though it was not stated there). In Section 4, we represent a slightly modified version of GMWZ1 treating this two-dimensional case, or more generally, cases with (H5) in a more direct way. Once these low-frequency resolvent bounds are obtained, the stability analysis follows in a standard fashion [Z2, Z3, Z4]. See Section 1.6 for further discussions.

1.1. Equations and assumptions. We consider the general hyperbolic-parabolic system of conservation laws (1.1) in conserved variable $\tilde{U}$, with

$$
\tilde{U}=\left(\begin{array}{c}
\tilde{u}^{I} \\
\tilde{u}^{I I}
\end{array}\right), \quad B^{j k}=\left(\begin{array}{cc}
0 & 0 \\
b_{1}^{j k} & b_{2}^{j k}
\end{array}\right),
$$

$\tilde{u}^{I} \in \mathbb{R}^{n-r}, \tilde{u}^{I I} \in \mathbb{R}^{r}$, and

$$
\Re \sigma \sum_{j k} b_{2}^{j k} \xi_{j} \xi_{k} \geq \theta|\xi|^{2}>0, \quad \forall \xi \in \mathbb{R}^{n} \backslash\{0\} .
$$

Following [Z3, Z4], we assume that equations (1.1) can be written, alternatively, after a triangular change of coordinates

$$
\tilde{W}:=\tilde{W}(\tilde{U})=\left(\begin{array}{c}
\tilde{w}^{I}\left(\tilde{u}^{I}\right) \\
\tilde{w}^{I I}\left(\tilde{u}^{I}, \tilde{u}^{I I}\right)
\end{array}\right),
$$

in the quasilinear, partially symmetric hyperbolic-parabolic form

$$
\tilde{A}^{0} \tilde{W}_{t}+\sum_{j} \tilde{A}^{j} \tilde{W}_{x_{j}}=\sum_{j k}\left(\tilde{B}^{j k} \tilde{W}_{x_{k}}\right)_{x_{j}}+\tilde{G}
$$

where

$$
\tilde{A}^{0}=\left(\begin{array}{cc}
\tilde{A}_{11}^{0} & 0 \\
0 & \tilde{A}_{22}^{0}
\end{array}\right), \quad \tilde{A}^{1}=\left(\begin{array}{cc}
\tilde{A}_{11}^{1} & \tilde{A}_{12}^{1} \\
\tilde{A}_{21}^{1} & \tilde{A}_{22}^{1}
\end{array}\right), \quad \tilde{B}^{j k}=\left(\begin{array}{cc}
0 & 0 \\
0 & \tilde{b}^{j k}
\end{array}\right)
$$

and, defining $\tilde{W}_{ \pm}:=\tilde{W}\left(U_{ \pm}\right)$,

(A1) $\tilde{A}^{j}\left(\tilde{W}_{ \pm}\right), \tilde{A}^{0}, \tilde{A}_{11}^{1}$ are symmetric, $\tilde{A}^{0} \geq \theta_{0}>0$,

(A2) for each $\xi \in \mathbb{R}^{d} \backslash\{0\}$, no eigenvector of $\sum_{j} \xi_{j} \tilde{A}^{j}\left(\tilde{A}^{0}\right)^{-1}\left(\tilde{W}_{ \pm}\right)$lies in the kernel of $\sum_{j k} \xi_{j} \xi_{k} \tilde{B}^{j k}\left(\tilde{A}^{0}\right)^{-1}\left(\tilde{W}_{ \pm}\right)$

(A3) $\Re \sigma \sum \tilde{b}^{j k} \xi_{j} \xi_{k} \geq \theta|\xi|^{2}$, and $\tilde{G}=\left(\begin{array}{l}0 \\ \tilde{g}\end{array}\right)$ with $\tilde{g}\left(\tilde{W}_{x}, \tilde{W}_{x}\right)=\mathcal{O}\left(\left|\tilde{W}_{x}\right|^{2}\right)$.

Along with the above structural assumptions, we make the following technical hypotheses: 
(H0) $F^{j}, B^{j k}, \tilde{A}^{0}, \tilde{A}^{j}, \tilde{B}^{j k}, \tilde{W}(\cdot), \tilde{g}(\cdot, \cdot) \in C^{s+1}$, for $s \geq[(d-1) / 2]+2$ in our analysis of linearized stability, and $s \geq s(d):=[(d-1) / 2]+4$ in our analysis of nonlinear stability.

(H1) The eigenvalues of $\tilde{A}_{11}^{1}$ are (i) distinct from the shock speed $s=0$; (ii) of common sign; and (iii) of constant multiplicity with respect to $U$.

(H2) $\operatorname{det}\left(d F^{1}\left(U_{ \pm}\right)\right) \neq 0$.

(H3) Local to $\bar{U}(\cdot)$, stationary solutions of (1.1), connecting $U_{ \pm}$, form a smooth manifold $\left\{\bar{U}^{\delta}(\cdot)\right\}, \delta \in \mathcal{U} \subset \mathbb{R}^{l}$.

(H4) The eigenvalues of $\sum_{j} \xi_{j} d F^{j}\left(U_{ \pm}\right)$have constant multiplicity with respect to $\xi \in \mathbb{R}^{d}$, $\xi \neq 0$.

Structural assumptions (A1)-(A3) and (H0)-(H2) are satisfied for gas dynamics and MHD; see discussions in [MaZ4, Z3, Z4, GMWZ5, GMWZ6].

Alternative Hypothesis $\mathbf{H} 4^{\prime}$. The constant multiplicity condition in Hypothesis (H4) holds for the compressible Navier Stokes equations whenever is hyperbolic. However, the condition fails always for the equations of viscous MHD. In the paper, we are able to treat symmetric systems like the viscous MHD under the following relaxed hypothesis.

(H4') The eigenvalues of $\sum_{j} \xi_{j} d F^{j}\left(U_{ \pm}\right)$are either semisimple and of constant multiplicity or totally nonglancing in the sense of [GMWZ6], Definition 4.3.

Remark 1.1. There will be easily seen that our results also apply to the case where the characteristic roots satisfy a (BS) condition! 1 (see Definition 4.9, [GMWZ6]), a more general situation than the constant multiplicity condition, ensuring that a suitable generalized block structure condition is satisfied. See Remark 2.3 for further discussion.

Remark 1.2. Here we stress that we are able to drop the following structural assumption, which is needed for the analyses of [Z2, Z3, Z4, GMWZ1].

(H5) The set of branch points of the eigenvalues of $\left(\tilde{A}^{1}\right)^{-1}\left(i \tau \tilde{A}^{0}+\sum_{j \neq 1} i \xi_{j} \tilde{A}^{j}\right)_{ \pm}, \tau \in \mathbb{R}$, $\tilde{\xi} \in \mathbb{R}^{d-1}$ is the (possibly intersecting) union of finitely many smooth curves $\tau=\eta_{q}^{ \pm}(\tilde{\xi})$, on which the branching eigenvalue has constant multiplicity $s_{q}$ (by definition $\geq 2$ ).

1.2. Shock profiles. We recall the following classification of shock profiles.

Hyperbolic Classification. Let $i_{+}$denote the dimension of the stable subspace of $d F^{1}\left(U_{+}\right), i_{-}$denote the dimension of the unstable subspace of $d F^{1}\left(U_{-}\right)$, and $i:=i_{+}+i_{-}$. Indices $i_{ \pm}$count the number of incoming characteristics from the right/left of the shock, while $i$ counts the total number of incoming characteristics toward the shock. Then, the hyperbolic classification of profile $\bar{U}(\cdot)$, i.e., the classification of the associated hyperbolic shock $\left(U_{-}, U_{+}\right)$, is

$$
\begin{cases}\text { Lax type } & \text { if } i=n+1 \\ \text { Undercompressive } & \text { if } i \leq n \\ \text { Overcompressive } & \text { if } i \geq n+2\end{cases}
$$

\footnotetext{
${ }^{1}$ Thanks to one of the referees for his pointing out this extension.
} 
In case all characteristics are incoming on one side, i.e. $i_{+}=n$ or $i_{-}=n$, a shock is called extreme.

Viscous Classification. A complete description of the viscous connection requires the further compressibility index $l$, where $l$ is defined as in (H3). In case the connection is "maximally" transverse:

$$
l=\left\{\begin{array}{lr}
1 & \text { Lax or undercompressive case } \\
i-n & \text { overcompressive case }
\end{array}\right.
$$

we call the shock "pure" type, and classify it according to its hyperbolic type. Otherwise, we call it "mixed" under/overcompressive type. Throughout this paper, we assume all viscous profiles are of pure, hyperbolic type.

For further discussions, see [Z2, Section 1.2] or [Z3, Section 1.2], and the references therein.

1.3. The uniform Evans stability condition. The linearized equations of (1.1) about $\bar{U}$ are

$$
U_{t}=L U:=\sum_{j, k}\left(B^{j k} U_{x_{k}}\right)_{x_{j}}-\sum_{j}\left(A^{j} U\right)_{x_{j}}
$$

with initial data $U(0)=U_{0}$. Here, $B^{j k}:=B^{j k}\left(\bar{U}\left(x_{1}\right)\right)$ and $A^{j} U:=d F^{j}\left(\bar{U}\left(x_{1}\right)\right) U$ $\left[d B^{j 1}\left(\bar{U}\left(x_{1}\right)\right) U\right] \bar{U}_{x_{1}}\left(x_{1}\right)$.

A necessary condition for linearized stability is weak spectral stability, defined as nonexistence of unstable spectra $\Re \lambda>0$ of the linearized operator $L$ about the wave. As described in [Z2, Z3], this is equivalent to nonvanishing for all $\tilde{\xi} \in \mathbb{R}^{d-1}, \Re \lambda>0$ of the Evans function

$$
D_{L}(\tilde{\xi}, \lambda)
$$

(see equation (A.3) in Appendix A) a Wronskian associated with the Fourier-transformed eigenvalue ODE. Let $\zeta=(\tilde{\xi}, \lambda)$. Introduce polar coordinates $\zeta=\rho \hat{\zeta}$, with $\hat{\zeta}=(\hat{\tilde{\xi}}, \hat{\lambda}) \in S^{d}$. We also define $S_{+}^{d}=S^{d} \bigcap\{\Re e \hat{\lambda} \geq 0\}$.

Definition 1.3. We define strong spectral stability as uniform Evans stability:

(D) $D_{L}(\hat{\zeta}, \rho)$ vanishes to precisely $l^{\text {th }}$ order at $\rho=0$ for all $\hat{\zeta} \in S_{+}^{d}$ and has no other zeros in $S_{+}^{d} \times \overline{\mathbb{R}}_{+}$, where $l$ is the compressibility index defined as in (H3) and (1.6).

The spectral stability of arbitrary-amplitude shocks can be checked efficiently by numerical Evans computations as in HLyZ1, HLyZ2.

1.4. The GMWZ result. We recall the recent result of Guès, Métivier, Williams, and Zumbrun for low-frequency regimes, and refer the reader to their original papers for the detail of statements and the proof.

Theorem 1.4 (GMWZ6], Theorems 3.7 and 3.9; GMWZ1], Section 8). Assume (A1)(A3), (HO)-(H3), and (H4').

Then, the strong spectral stability condition $(D)$ implies the $L^{2}$ uniform stability estimate for low-frequency regimes (precisely stated below, (2.13), Section 2.2). 
Example 1.5 ([GMWZ6], Section 8). Fast Lax' shocks for viscous MHD equations satisfy the structural assumptions of Theorem 1.4.

However, it is also shown that

Counterexample 1.6 (GMWZ6], Section 8). Slow Lax' shocks for viscous MHD equations do not satisfy the structural assumption (H4'), and thus Theorem 1.4 does not apply to these cases.

1.5. Main results. Our main results are as follows.

Theorem 1.7 (Linearized stability). Assuming (A1)-(A3), (HO)-(H3), (H4'), and (D), we obtain the asymptotic $L^{1} \cap H^{[(d-1) / 2]+2} \rightarrow L^{p}$ stability of (1.7) for all three types of shocks in dimensions $d \geq 3$, for any $2 \leq p \leq \infty$, with rates of decay

$$
\begin{aligned}
& |U(t)|_{L^{2}} \leq C(1+t)^{-\frac{d-2}{4}}\left|U_{0}\right|_{L^{1} \cap L^{2}}, \\
& |U(t)|_{L^{p}} \leq C(1+t)^{-\frac{d-1}{2}(1-1 / p)+\frac{1}{4}}\left|U_{0}\right|_{L^{1} \cap H^{[(d-1) / 2]+2},}
\end{aligned}
$$

provided that the initial perturbations $U_{0}$ are in $L^{1} \cap L^{2}$ for $p=2$, or in $L^{1} \cap H^{[(d-1) / 2]+2}$ for $p>2$.

Theorem 1.8 (Nonlinear stability). Assuming (A1)-(A3), (H0)-(H3), (H4'), and (D), we obtain the asymptotic $L^{1} \cap H^{s} \rightarrow L^{p} \cap H^{s}$ stability for Lax or overcompressive shocks in dimension $d \geq 3$ and undercompressive shocks in dimensions $d \geq 5$, for $s \geq s(d)$ as defined in (HO), and any $2 \leq p \leq \infty$, with rates of decay

$$
\begin{aligned}
& |\tilde{U}(t)-\bar{U}|_{L^{p}} \leq C(1+t)^{-\frac{d-1}{2}(1-1 / p)+\frac{1}{4}}\left|U_{0}\right|_{L^{1} \cap H^{s}} \\
& |\tilde{U}(t)-\bar{U}|_{H^{s}} \leq C(1+t)^{-\frac{d-2}{4}}\left|U_{0}\right|_{L^{1} \cap H^{s}},
\end{aligned}
$$

provided that the initial perturbations $U_{0}:=\tilde{U}_{0}-\bar{U}$ are sufficiently small in $L^{1} \cap H^{s}$.

Remark 1.9. The price of dropping Hypothesis (H5) is that the obtained rate of decay is degraded by $t^{1 / 4}$ as comparing to those established in [Z2, [Z3, [Z4] or Theorem [1.10 below. Therefore the rates are possibly not sharp. In fact, we believe that the sharp rate of decay in $L^{2}$ is rather that of a $d$-dimensional heat kernel and the sharp rate of decay in $L^{\infty}$ dependent on the characteristic structure of the associated inviscid equations, as in the constant-coefficient case HoZ1, HoZ2.

Our next main result addresses the stability for the two-dimensional case that is not covered by the above theorems. We remark here that as shown in [Z3], page 321, Hypothesis (H5) is automatically satisfied in dimensions $d=1,2$ and in any dimension for rotationally invariant problems. Thus, in treating the two-dimensional case, we assume this hypothesis without making any further restriction on structure of the systems. Also since it turns out that the proof does not depend on the dimensions, we state (and prove) the theorem in a general form as follows, recovering previous results of K. Zumbrun (see [Z3, Theorem 5.5]) for "uniformly inviscid stable" Lax or over-compressive shocks with same decay rates. 
Theorem 1.10 (Two-dimensional case or cases with (H5)). Assume the same hypotheses as in Theorems 1.7 and 1.8 with additional assumption (H5). Then Lax or over-compressive shocks are asymptotically nonlinearly $L^{1} \cap H^{s} \rightarrow L^{p} \cap H^{s}$ stable in dimensions $d \geq 2$, for any $2 \leq p \leq \infty$, with rates of decay

$$
\begin{aligned}
|\tilde{U}(t)-\bar{U}|_{L^{p}} & \leq C(1+t)^{-\frac{d-1}{2}(1-1 / p)}\left|U_{0}\right|_{L^{1} \cap H^{s}} \\
|\tilde{U}(t)-\bar{U}|_{H^{s}} & \leq C(1+t)^{-\frac{d-1}{4}}\left|U_{0}\right|_{L^{1} \cap H^{s}},
\end{aligned}
$$

provided that the initial perturbations $U_{0}:=\tilde{U}_{0}-\bar{U}$ are sufficiently small in $L^{1} \cap H^{s}$. Similar statement can be stated for linearized stability with same decay rates.

1.6. Discussion and open problems. As observed in [Z3, $\mathrm{Z4}$, the high-frequency estimate on the solution operator has already been established without the structural assumptions (H4)-(H5), mainly relying on the damping energy estimates. Hence we shall use it here as a black box. We would like to draw the reader's attention to our recent work in [NZ2] for a great simplification of this original high-frequency argument, requiring higher regularity of the forcing $f$ (to credit, the simplification was based on an argument introduced in [KZ] for relaxation shocks).

The difficulty of relaxing Hypothesis (H4) and dropping (H5), extending results in [Z2, [Z3, Z4 obtained by pointwise bound approach, is that there and in GMWZ1 the authors apply the diagonalization of glancing blocks, where the hypotheses are required, to obtain rather sharp bounds on resolvent kernel and resolvent solution. We rather use the $L^{2}$ stability bound more directly, avoiding to get sharp bounds on the adjoint problem where the diagonalization of glancing blocks must be applied (see Section 12, GMWZ1]), and as a consequence, avoiding the diagonalization error (denoted by $\beta$ in [GMWZ1] or $\gamma_{2}$ in [Z3]) at the expense of slightly degraded decay, comparing to those reported in [Z2, Z3, GMWZ1]. However, the loss $t^{1 / 4}$ of decay is still sufficient to close our analysis for dimensions $d \geq 3$ in the Lax or overcompressive case and for $d \geq 5$ in the undercompressive case. As already mentioned at the beginning of the paper, this $L^{1} \rightarrow L^{p}$ resolvent bound will be the key to the improvement.

Our analysis indeed applies to all applications covered by the GMWZ small viscosity theory. Hence, the remaining open problem is to treat cases that are not covered by the GMWZ theory, that is, the cases when the structural assumption (H4') of Theorem 1.4 is not satisfied or more generally when the generalized block structure fails. Counterexample 1.6 is showing one of such interesting but untreated cases, violating the structural assumption (H4').

It is also worth mentioning that the undercompressive shock analysis was carried out in [Z3] only in nonphysical dimensions $d \geq 4$, and thus still remains open in dimensions for $d \leq 3$ for systems with or without assumptions (H4)-(H5). Finally, in our forthcoming paper [N2], we have been able to carry out the analysis for boundary layers in dimensions $d \geq 2$, extending our recent results in [NZ2] to systems with variable multiplicities. It turns out that the analysis for the boundary layer case is quite more delicate than those for the case of Lax or overcompressive shocks that we are studying here. 


\section{LineARIZED ESTIMATES}

The linearized equations of (1.1) about the profile $\bar{U}$ are

$$
U_{t}=L U:=\sum_{j, k}\left(B^{j k} U_{x_{k}}\right)_{x_{j}}-\sum_{j}\left(A^{j} U\right)_{x_{j}}
$$

with initial data $U(0)=U_{0}$.

Then, we obtain the following proposition.

Proposition 2.1. Under the hypotheses of Theorems 1.7 and 1.8, the solution operator $\mathcal{S}(t):=e^{L t}$ of the linearized equations may be decomposed into low frequency and high frequency parts (defined precisely below) as $\mathcal{S}(t)=\mathcal{S}_{1}(t)+\mathcal{S}_{2}(t)$ satisfying

$$
\left|\mathcal{S}_{1}(t) \partial_{x_{1}}^{\beta_{1}} \partial_{\tilde{x}}^{\tilde{\beta}} f\right|_{L_{x}^{p}} \leq C(1+t)^{-\frac{d-1}{2}(1-1 / p)+\frac{1}{4}-\frac{|\tilde{\beta}|}{2}-(1-\alpha) \frac{\beta_{1}}{2}}|f|_{L_{x}^{1}}
$$

for all $2 \leq p \leq \infty, d \geq 3$, and $\beta=\left(\beta_{1}, \tilde{\beta}\right)$ with $\beta_{1}=0,1$ and $\alpha$ defined as

$$
\alpha:= \begin{cases}0 & \text { for Lax or overcompressive case } \\ 1 & \text { for undercompressive case }\end{cases}
$$

and

$$
\left|\partial_{x_{1}}^{\gamma_{1}} \partial_{\tilde{x}}^{\tilde{\gamma}} \mathcal{S}_{2}(t) f\right|_{L^{2}} \leq C e^{-\theta_{1} t}|f|_{H^{\left|\gamma_{1}\right|+|\tilde{\gamma}|}},
$$

for $\gamma=\left(\gamma_{1}, \tilde{\gamma}\right)$ with $\gamma_{1}=0,1$.

Here, we use the same decomposition of solution operator $\mathcal{S}(t)$ as in the article of $\mathrm{K}$. Zumbrun [Z3]; see (5.152)-(5.153) in [Z3] or (2.32) below.

2.1. High-frequency estimate. We observe that our relaxed Hypothesis (H4') and the dropped Hypothesis (H5) only play a role in low-frequency regimes. Thus, in course of obtaining the high-frequency estimate (2.4), we make here the same assumptions as were made in [Z3], and therefore the same estimate remains valid as claimed in (2.4) under our current assumptions. We omit to repeat its proof here, and refer the reader to the article [Z3], (5.16), Proposition 5.7, for the original proof. See also a great simplification in [NZ2], Proposition 3.6 in treating the boundary layer case.

In the remaining of this section, we shall focus on proving the bounds on low-frequency part $\mathcal{S}_{1}(t)$ of the linearized solution operator.

Taking the Fourier transform in $\tilde{x}:=\left(x_{2}, \ldots, x_{d}\right)$ of linearized equation (2.1), we obtain a family of eigenvalue ODE

$$
\begin{aligned}
\lambda U=L_{\tilde{\xi}} U:=\overbrace{\left(B_{11} U^{\prime}\right)^{\prime}-\left(A_{1} U\right)^{\prime}}^{L_{0} U}-i \sum_{j \neq 1} A_{j} \xi_{j} U+i \sum_{j \neq 1} B_{j 1} \xi_{j} U^{\prime} \\
+i \sum_{k \neq 1}\left(B_{1 k} \xi_{k} U\right)^{\prime}-\sum_{j, k \neq 1} B_{j k} \xi_{j} \xi_{k} U .
\end{aligned}
$$


2.2. $L^{2}$ stability estimate for low frequencies. We briefly recall the procedure (see GMWZ1, page 75-85) of reducing the eigenvalue equations to the block structure equations and stating the $L^{2}$ estimate for low-frequency regimes by the construction of degenerate symmetrizers.

Let $U=\left(u^{I}, u^{I I}\right)^{T}$ a solution of eigenvalue equations, that is, $\left(L_{\tilde{\xi}}-\lambda\right) U=f$ where $L_{\tilde{\xi}}$ is defined as in (2.5). Following [Z3, Section 2.4], consider the variable $W$ as usual

$$
W:=\left(\begin{array}{c}
u^{I} \\
u^{I I} \\
z
\end{array}\right)
$$

with $z:=b_{1}^{11} u_{x_{1}}^{I}+b_{2}^{11} u_{x_{1}}^{I I}$. Then we can write equations of $W$ as a first order system

$$
\partial_{x_{1}} W=\mathcal{G}\left(x_{1}, \lambda, \tilde{\xi}\right) W+F,
$$

with $F:=\left(A_{*}^{-1} f^{I}, 0, f^{I I}\right)^{t r}$, where $f=\left(f^{I}, f^{I I}\right)^{t r}$ and $A_{*}:=A_{11}^{1}-A_{12}^{1}\left(b_{2}^{11}\right)^{-1} b_{1}^{11}$; thus, in particular, $|F| \leq C|f|$, for some constant $C$. It is not necessary for us to carry out in detail the form of $\mathcal{G}$; though, see equation (2.65) of [Z3]. Indeed, we are only interested in the fact that bounds in $L^{p}$ of $W$ will give those of $U$ in the same norm.

We go further as in GMWZ1, page 75] to write this $(n+r) \times(n+r)$ system on $\mathbb{R}$ as an equivalent $2(n+r) \times 2(n+r)$ "doubled" boundary problem on $x_{1} \geq 0$ :

$$
\begin{aligned}
& \partial_{x_{1}} \tilde{W}=\tilde{\mathcal{G}}\left(x_{1}, \lambda, \tilde{\xi}\right) \tilde{W}+\tilde{F} \\
& \Gamma \tilde{W}=0 \text { on } x_{1}=0
\end{aligned}
$$

where

$$
\begin{aligned}
& \tilde{W}\left(x_{1}, \lambda, \tilde{\xi}\right)=\left(W_{+}, W_{-}\right), \\
& \tilde{\mathcal{G}}\left(x_{1}, \lambda, \tilde{\xi}\right)=\left(\begin{array}{cc}
\mathcal{G}_{+} & 0 \\
0 & -\mathcal{G}_{-}
\end{array}\right), \\
& \tilde{F}=\left(\begin{array}{c}
F_{+} \\
-F_{-}
\end{array}\right), \\
& \Gamma \tilde{W}=W_{+}-W_{-}
\end{aligned}
$$

with $F_{ \pm}\left(x_{1}\right):=F\left( \pm x_{1}\right)$.

For small or bounded frequencies $(\lambda, \tilde{\xi})$, we use the known MZ conjugation; see, for example, [MeZ1] or [GMWZ1, Lemma 5.1]. That is, given any $(\underline{\lambda}, \underline{\tilde{\xi}}) \in \mathbb{R}^{d+1}$, there is a smooth invertible matrix $\Phi\left(x_{1}, \lambda, \tilde{\xi}\right)$ for $x_{1} \geq 0$ and $(\lambda, \tilde{\xi})$ in a small neighborhood of $(\underline{\lambda}, \underline{\tilde{\xi}})$, such that (2.7) is equivalent to

$$
\partial_{x_{1}} Y=\mathcal{G}_{+}(\lambda, \tilde{\xi}) Y+\tilde{\tilde{F}}, \quad \tilde{\Gamma}(\lambda, \tilde{\xi}) Y=0
$$

where $\mathcal{G}_{+}(\lambda, \tilde{\xi}):=\tilde{\mathcal{G}}(+\infty, \lambda, \tilde{\xi}), \tilde{W}=\Phi Y, \tilde{\tilde{F}}=\Phi^{-1} \tilde{F}$ and $\tilde{\Gamma} Y:=\Gamma \Phi Y$.

Next, there are smooth matrices $V(\lambda, \tilde{\xi})$ such that

$$
V^{-1} \mathcal{G}_{+} V=\left(\begin{array}{cc}
H & 0 \\
0 & P
\end{array}\right)
$$


with blocks $H(\lambda, \tilde{\xi})$ and

$$
P(\lambda, \tilde{\xi})=\left(\begin{array}{cc}
P_{+} & 0 \\
0 & P_{-}
\end{array}\right)
$$

satisfying the eigenvalues $\mu$ of $P_{ \pm}$in $\{ \pm \Re e \mu \geq c>0\}$ and

$$
\begin{aligned}
H(\lambda, \tilde{\xi}) & =H_{0}(\lambda, \tilde{\xi})+\mathcal{O}\left(\rho^{2}\right) \\
H_{0}(\lambda, \tilde{\xi}): & =-\left(A_{+}^{1}\right)^{-1}\left((i \tau+\gamma) A_{+}^{0}+\sum_{j=2}^{d} i \xi_{j} A_{+}^{j}\right) .
\end{aligned}
$$

Define variables $Z=\left(u_{H}, u_{P}\right)^{T}$ with $u_{P}:=\left(u_{P_{+}}, u_{P_{-}}\right)^{T}$ as

$$
\tilde{W}=\Phi Y=\Phi V Z, \quad \bar{\Gamma} Z:=\Gamma \Phi V Z,
$$

and $\left(F_{H}, F_{P}\right)^{T}=V^{-1} \tilde{\tilde{F}}$. We have

$$
\partial_{x_{1}}\left(\begin{array}{c}
u_{H} \\
u_{P_{ \pm}}
\end{array}\right)=\left(\begin{array}{cc}
H & 0 \\
0 & P_{ \pm}
\end{array}\right)\left(\begin{array}{c}
u_{H} \\
u_{P_{ \pm}}
\end{array}\right)+\left(\begin{array}{c}
F_{H} \\
F_{P_{ \pm}}
\end{array}\right), \quad \bar{\Gamma} Z=0 .
$$

Let $\langle\cdot, \cdot\rangle$ denote the standard $L^{2}$ product over $[0, \infty)$, that is,

$$
\langle f, g\rangle=\int_{0}^{\infty} f\left(x_{1}\right) \bar{g}\left(x_{1}\right) d x_{1}, \quad \forall f, g \in L^{2}(0, \infty),
$$

where $\bar{g}$ is the complex conjugate of $g$.

Then, recalling that $\rho=|(\tilde{\xi}, \lambda)|$ and $\gamma=\Re e \lambda$, we obtain the maximal stability estimate for the low frequency regimes ([GMWZ6, GMWZ1]):

$$
\begin{aligned}
\left(\gamma+\rho^{2}\right)\left|u_{H}\right|_{L^{2}}^{2}+\left|u_{P_{+}}\right|_{L^{2}}^{2} & +\rho^{2}\left|u_{P_{-}}\right|_{L^{2}}^{2}+\left|u_{H}(0)\right|^{2}+\left|u_{P_{+}}(0)\right|^{2}+\rho^{2}\left|u_{P_{-}}(0)\right|^{2} \\
& \lesssim\left|\left\langle S F_{P_{+}}, u_{P_{+}}\right\rangle\right|+\left|\left\langle S F_{P_{-}}, u_{P_{-}}\right\rangle\right|+\left|\left\langle S F_{H}, u_{H}\right\rangle\right|
\end{aligned}
$$

where $S$ is the degenerate symmetrizer constructed in [GMWZ1] (see equations (8.2),(8.2), and (6.18)) as follows

$$
S=\left(\begin{array}{cc}
S_{P} & 0 \\
0 & S_{H}
\end{array}\right)
$$

and

$$
S_{P}=\left(\begin{array}{cc}
K I d & 0 \\
0 & -\rho^{2}
\end{array}\right)
$$

for sufficiently large constant $K$ (independent of small parameter $\rho$ ); here, $I d$ is the identity matrix and the two subblocks in $S_{P}$ have the same sizes as those of $P_{ \pm}$in (2.11), correspondingly. Here and throughout the paper, by $f \lesssim g$, we mean $f \leq C g$, for some positive constant $C$ independent of $\rho$.

There are two possibly subtle points in quoting (2.13) that we would like to point out, namely, (i) the estimate (2.13) was proved in Section 8, GMWZ1, under the assumption (H4), but not under the relaxed Hypothesis (H4'), and (ii) the estimate was obtained only for the Lax shock case. However, in the first matter, the variable multiplicity assumption is only involved in the hyperbolic part (the $H$ block in (2.12) ) and the parabolic blocks $P_{ \pm}$remain the same. Thus, the degenerate Kreiss-type symmetrizers techniques (only 
involved in the parabolic blocks) introduced in [GMWZ1] can still be applicable here. For the hyperbolic part, we now use the recent construction of Kreiss-type symmetrizers in GMWZ6 that applies to the relaxed Hypothesis (H4'), thus yielding the $L^{2}$ estimate for this block. In dealing with the second matter, we recall that a crucial step in the analysis of [GMWZ1] for the Lax shock case was to proving the "right" degeneracy of the boundary operator or Lemma 7.1 in GMWZ1, connecting with the Evans stability condition (D). We then observe that with slight modification of the proof, the lemma remains unchanged for the under/over-compressive shock case, yielding the same result. For sake of completeness, we shall recall the proof of Lemma 7.1, GMWZ1, with a straightforward extension to other cases than the Lax case in Appendix A.

In other words, with our above observations, we may use the estimate (2.13) as stated under our current assumptions in treating all three types of shocks. In addition, thanks to that fact that the symmetrizer $S$ is degenerate with order $\rho^{2}$ in the block $P_{-}$(see (2.14), (2.15) above), we can further estimate (2.13) as

$$
\begin{aligned}
\left(\gamma+\rho^{2}\right)\left|u_{H}\right|_{L^{2}}^{2}+\left|u_{P_{+}}\right|_{L^{2}}^{2} & +\rho^{2}\left|u_{P_{-}}\right|_{L^{2}}^{2}+\left|u_{H}(0)\right|^{2}+\left|u_{P_{+}}(0)\right|^{2}+\rho^{2}\left|u_{P_{-}}(0)\right|^{2} \\
& \lesssim\left\langle\left|F_{P_{+}}\right|,\left|u_{P_{+}}\right|\right\rangle+\rho^{2}\left\langle\left|F_{P_{-}}\right|,\left|u_{P_{-}}\right|\right\rangle+\left\langle\left|F_{H}\right|,\left|u_{H}\right|\right\rangle .
\end{aligned}
$$

We note that in a final step in [GMWZ1, equation (8.11)], the standard Young's inequality was used to absorb all terms of $\left(u_{H}, u_{P}\right)$ into the left-hand side, leaving the $L^{2}$ norm of $F$ alone in the right hand side. For our purpose, we shall keep it as stated in (2.16).

We remark also that as shown in GMWZ1, all of coordinate transformation matrices are uniformly bounded. Thus a bound on $Z=\left(u_{H}, u_{P}\right)^{T}$ would yield a corresponding bound on the solution $U$.

2.3. $L^{1} \rightarrow L^{p}$ estimates. We establish the $L^{1} \rightarrow L^{p}$ resolvent bounds for solutions of eigenvalue equations $\left(L_{\tilde{\xi}}-\lambda\right) U=f$ in the low frequency regime; specifically, we are interested in regime of parameters restricting to the surface

$$
\Gamma^{\tilde{\xi}}:=\left\{\lambda: \Re e \lambda=-\theta_{1}\left(|\tilde{\xi}|^{2}+|\Im m \lambda|^{2}\right)\right\},
$$

for $\theta_{1}>0$ and $|(\tilde{\xi}, \lambda)|$ sufficiently small. The curve $\Gamma^{\tilde{\xi}}$ was introduced in [Z2, equation (4.26)]. Introducing $\Gamma^{\tilde{\xi}}$ is in fact regarded as a key to the analysis of long-time stability in multidimensions. The main point here is that even though $\lambda$ enters into the stable complex half-plane $(\{\Re e \lambda<0\}), \Gamma^{\tilde{\xi}}$ remains outside of the essential spectrum of limiting linearized operators $L_{\tilde{\xi}, \pm}$; see [Z3, Lemma 2.21].

In addition, in a related matter, we would like to recall that the Kreiss' symmetrizers constructed by O. Guès, G. Métivier, M. Williams, and K. Zumbrun can be attained in a full neighborhood of basepoint $(\underline{\xi}, \underline{\lambda})$ even for $\Re \underline{\lambda}=0$ (see, e.g., Theorem 3.7, GMWZ6]). Thus, the $L^{2}$ estimate (2.16) is in fact still valid in any region of

$$
\gamma \geq-\theta\left(|\tau|^{2}+|\tilde{\xi}|^{2}\right)
$$

for $\theta$ sufficiently small. In particular, we shall use (2.16) for $\lambda$ restricted on the curve $\Gamma^{\tilde{\xi}}$.

We obtain the following: 
Proposition 2.2 (Low-frequency bounds). Under the hypotheses of Theorem 1.8, for $\lambda \in$ $\Gamma^{\tilde{\xi}}$ and $\rho:=|(\tilde{\xi}, \lambda)|, \theta_{1}$ sufficiently small, there holds the resolvent bound

$$
\left|\left(L_{\tilde{\xi}}-\lambda\right)^{-1} \partial_{x_{1}}^{\beta} f\right|_{L^{p}\left(x_{1}\right)} \leq C \rho^{-3 / 2+(1-\alpha) \beta}|f|_{L^{1}\left(x_{1}\right)},
$$

for all $2 \leq p \leq \infty, \beta=0,1$, and $\alpha$ defined as in (2.3).

Proof. Changing variables as above subsection and taking the inner product of each equation in (2.12) against $u_{H}$ and $u_{P_{ \pm}}$, respectively, and integrating the results over $\left[0, x_{1}\right]$, for $x_{1}>0$, we obtain

$$
\begin{aligned}
\frac{1}{2}\left|u_{H}\left(x_{1}\right)\right|^{2} & =\frac{1}{2}\left|u_{H}(0)\right|^{2}+\Re e \int_{0}^{x_{1}}\left(H(\lambda, \tilde{\xi}) u_{H} \cdot u_{H}+F_{H} \cdot u_{H}\right) d z \\
\frac{1}{2}\left|u_{P_{ \pm}}\left(x_{1}\right)\right|^{2} & =\frac{1}{2}\left|u_{P_{ \pm}}(0)\right|^{2}+\Re e \int_{0}^{x_{1}}\left(P_{ \pm}(\lambda, \tilde{\xi}) u_{P_{ \pm}} \cdot u_{P_{ \pm}}+F_{P_{ \pm}} \cdot u_{P_{ \pm}}\right) d z
\end{aligned}
$$

This together with use of Young's inequality into the last terms involved in $F$ and the facts that $|H| \leq C \rho$ and $\left|P_{ \pm}\right| \leq C$ yields

$$
\begin{aligned}
\left|u_{H}\right|_{L^{\infty}\left(x_{1}\right)}^{2} & \lesssim\left|u_{H}(0)\right|^{2}+\rho\left|u_{H}\right|_{L^{2}}^{2}+\left|F_{H}\right|_{L^{1}}^{2}, \\
\left|u_{P_{ \pm}}\right|_{L^{\infty}\left(x_{1}\right)}^{2} & \lesssim\left|u_{P_{ \pm}}(0)\right|^{2}+\left|u_{P_{ \pm}}\right|_{L^{2}}^{2}+\left|F_{P_{ \pm}}\right|_{L^{1}}^{2} .
\end{aligned}
$$

We are now in position of applying the $L^{2}$ stability estimate (2.16). In (2.20), multiplying both sides of equations of $u_{H}$ by $\rho$, of $u_{P_{+}}$by 1 , and of $u_{P_{-}}$by $\rho^{2}$, adding up results, and applying (2.16), we obtain

$$
\begin{aligned}
\rho^{2}\left(\left|u_{H}\right|_{L^{2}}^{2}\right. & \left.+\left|u_{P}\right|_{L^{2}}^{2}\right)+\rho\left|u_{H}\right|_{L^{\infty}}^{2}+\left|u_{P_{+}}\right|_{L^{\infty}}^{2}+\rho^{2}\left|u_{P_{-}}\right|_{L^{\infty}}^{2} \\
& \lesssim\left\langle\left|F_{P_{+}}\right|,\left|u_{P_{+}}\right|\right\rangle+\rho^{2}\left\langle\left|F_{P_{-}}\right|,\left|u_{P_{-}}\right|\right\rangle+\left\langle\left|F_{H}\right|,\left|u_{H}\right|\right\rangle+\left|F_{H}\right|_{L^{1}}^{2}+\left|F_{P}\right|_{L^{1}}^{2},
\end{aligned}
$$

(noting that $\rho$ is assumed to be small; in particular, $\rho \leq 1$.)

Applying again the standard Young's inequality:

$$
\begin{aligned}
& \left\langle\left|F_{H}\right|,\left|u_{H}\right|\right\rangle+\left\langle\left|F_{P_{+}}\right|,\left|u_{P_{+}}\right|\right\rangle+\rho^{2}\left\langle\left|F_{P_{-}}\right|,\left|u_{P_{-}}\right|\right\rangle \\
& \quad \lesssim \epsilon\left[\rho\left|u_{H}\right|_{L^{\infty}}^{2}+\left|u_{P_{+}}\right|_{L^{\infty}}^{2}+\rho^{2}\left|u_{P_{-}}\right|_{L^{\infty}}^{2}\right]+C_{\epsilon}\left[\rho^{-1}\left|F_{H}\right|_{L^{1}}^{2}+\left|F_{P_{+}}\right|_{L^{1}}^{2}+\rho^{2}\left|F_{P_{-}}\right|_{L^{1}}^{2}\right]
\end{aligned}
$$

with $\epsilon>0$ being sufficiently small, from (2.21), we easily arrive at

$$
\begin{aligned}
\rho^{2}\left(\left|u_{H}\right|_{L^{2}}^{2}\right. & \left.+\left|u_{P}\right|_{L^{2}}^{2}\right)+\rho\left|u_{H}\right|_{L^{\infty}}^{2}+\left|u_{P_{+}}\right|_{L^{\infty}}^{2}+\rho^{2}\left|u_{P_{-}}\right|_{L^{\infty}}^{2} \\
& \lesssim \rho^{-1}\left|F_{H}\right|_{L^{1}}^{2}+\left|F_{P_{+}}\right|_{L^{1}}^{2}+\rho^{2}\left|F_{P_{-}}\right|_{L^{1}}^{2}+\left|F_{H}\right|_{L^{1}}^{2}+\left|F_{P}\right|_{L^{1}}^{2} .
\end{aligned}
$$

Therefore in term of $Z=\left(u_{H}, u_{P}\right)^{t}$, simplifying the above yields

$$
\rho^{2}|Z|_{L^{2}\left(x_{1}\right)}^{2}+\rho^{2}|Z|_{L^{\infty}\left(x_{1}\right)}^{2} \leq C \rho^{-1}|F|_{L^{1}}^{2}
$$

Now from the change of variables $Z=V^{-1} \Phi^{-1} \tilde{W}$, we have the same estimates for $\tilde{W}$ and thus $U$, because all coordinate transformation matrices are uniformly bounded. Hence, we also obtain bounds (2.23) for $U$ or by the interpolation inequality:

$$
|U|_{L^{p}\left(x_{1}\right)} \leq C \rho^{-3 / 2}|f|_{L^{1}\left(x_{1}\right)} .
$$

This thus proves the proposition in the case of $\beta=0$. 
For $\beta=1$, we expect that $\partial_{x_{1}} f$ plays a role as " $\rho f$ " forcing. Recall that the eigenvalue equations $\left(L_{\tilde{\xi}}-\lambda\right) U=\partial_{x_{1}} f$ read

$$
\begin{aligned}
\overbrace{\left(B^{11} U_{x_{1}}\right)_{x_{1}}-\left(A^{1} U\right)_{x_{1}}}^{L_{0} U} & -i \sum_{j \neq 1} A^{j} \xi_{j} U+i \sum_{j \neq 1} B^{j 1} \xi_{j} U_{x_{1}} \\
& +i \sum_{k \neq 1}\left(B^{1 k} \xi_{k} U\right)_{x_{1}}-\sum_{j, k \neq 1} B^{j k} \xi_{j} \xi_{k} U-\lambda U=\partial_{x_{1}} f .
\end{aligned}
$$

Now modifying the nice argument of Kreiss-Kreiss presented in [KK, GMWZ1], we write $U=V+U_{1}$, where $V$ satisfies

$$
\left(L_{0}-\lambda_{0}\right) V=\partial_{x_{1}} f, \quad x_{1} \in \mathbb{R},
$$

for $\lambda_{0}=\rho$. Noting that $A^{1}$ and $B^{11}$ depend on $x_{1}$ only, we thus can apply here the onedimensional Green kernel bounds investigated by C. Mascia and K. Zumbrun as follows.

Let $G_{\lambda_{0}}^{0}$ be the Green kernel of $\lambda_{0}-L_{0}$. Observe that our assumptions as projected on one-dimensional situations (i.e., $\tilde{\xi}=0$ ) are still the same as those in [Z3]. Thus, we apply Proposition 4.22 in [Z3] for (2.26), noting that $\lambda_{0}=\rho$ is sufficiently small. After a simplification, we simply obtain

$$
\left|G_{\lambda_{0}}^{0}\left(x_{1}, y_{1}\right)\right| \leq C\left[\rho^{-1} e^{-\theta\left|x_{1}\right|} e^{-\rho\left|y_{1}\right|}+e^{-\rho\left|x_{1}-y_{1}\right|}\right],
$$

and

$$
\left|\partial_{y_{1}} G_{\lambda_{0}}^{0}\left(x_{1}, y_{1}\right)\right| \leq C\left[\rho^{-1} e^{-\theta\left|x_{1}\right|}\left(\rho e^{-\theta \rho\left|y_{1}\right|}+\alpha e^{-\theta\left|y_{1}\right|}\right)+e^{-\rho\left|x_{1}-y_{1}\right|}\left(\rho+\alpha e^{-\theta\left|y_{1}\right|}\right)\right],
$$

where $\alpha$ is defined as in (2.3). We would like to remark here that Lemma 5.23, [Z3], gives the estimate (2.28) with $\alpha=0$ only for the Lax or overcompressive shocks. For the undercompressive shocks, we must have the weaker bound by the term $e^{-\theta\left|y_{1}\right|}$, that is, $\alpha=1$ (for further discussion, see, e.g., equations below (5.106), [Z3]).

Hence, using (2.28) and applying the standard Hausdorff-Young's inequality, we obtain

$$
|V|_{L^{p}\left(x_{1}\right)}+\left|V_{x_{1}}\right|_{L^{p}\left(x_{1}\right)} \lesssim|f|_{L^{1}\left(x_{1}\right)}+\alpha \rho^{-1}|f|_{L^{1}\left(x_{1}\right)} \lesssim \rho^{-\alpha}|f|_{L^{1}\left(x_{1}\right)},
$$

for all $1 \leq p \leq \infty$ and $\alpha=0$ or 1 defined as in (2.3).

Now from $U_{1}=U-V$ and equations of $U$ and $V$, we observe that $U_{1}$ satisfies

$$
\left(L_{\tilde{\xi}}-\lambda\right) U_{1}=L\left(V, V_{x_{1}}\right),
$$

where

$$
\begin{aligned}
L\left(V, V_{x_{1}}\right):= & i \sum_{j \neq 1} A^{j} \xi_{j} V-i \sum_{j \neq 1} B^{j 1} \xi_{j} V_{x_{1}}-i \sum_{k \neq 1}\left(B^{1 k} \xi_{k} V\right)_{x_{1}} \\
& +\sum_{j, k \neq 1} B^{j k} \xi_{j} \xi_{k} V+\left(\lambda-\lambda_{0}\right) V \\
& =\rho \mathcal{O}\left(|V|+\left|V_{x_{1}}\right|\right) .
\end{aligned}
$$


Therefore applying the result which we just proved for $\beta=0$ to the equations (2.30), we obtain

$$
\begin{aligned}
\left|U_{1}\right|_{L^{p}\left(x_{1}\right)} & \leq C \rho^{-3 / 2}\left|L\left(V, V_{x_{1}}\right)\right|_{L^{1}\left(x_{1}\right)} \leq C \rho^{-3 / 2} \rho\left[|V|_{L^{1}}+\left|V_{x_{1}}\right|_{L^{1}}\right] \\
& \leq C \rho^{-3 / 2+(1-\alpha)}|f|_{L^{1}\left(x_{1}\right)} .
\end{aligned}
$$

Bounds (2.29), (2.31) on $V$ and $U_{1}$ clearly give our claimed bounds on $U$ by triangle inequality:

$$
|U|_{L^{p}} \leq|V|_{L^{p}}+\left|U_{1}\right|_{L^{p}}
$$

We thus obtain the proposition.

Remark 2.3. Under the general structural assumptions, our proof of the $L^{1} \rightarrow L^{p}$ bounds above depends only on the $L^{2}$ maximal estimate (2.16). As the GMWZ theory covers to a more general case than $\left(\mathrm{H} 4^{\prime}\right)$, namely, the (BS) condition (Definition 4.9, GMWZ6]), our results thus apply to this case as well without any additional work.

Remark 2.4. In Appendices B and C, we will prove a slightly-weaker resolvent estimate like (2.18) in which the Green kernel bounds (2.27), (2.28) will not be used. Thus, our main results can in fact be derived completely independent of the pointwise Green function estimates.

2.4. Estimates on the solution operator. In this subsection, we complete the proof of Proposition 2.1. As mentioned earlier, it suffices to prove the bounds for $\mathcal{S}_{1}(t)$, where the low frequency solution operator $\mathcal{S}_{1}(t)$ is defined as

$$
\mathcal{S}_{1}(t):=\frac{1}{(2 \pi i)^{d}} \int_{|\tilde{\xi}| \leq r} \oint_{\Gamma \tilde{\xi} \cap\{|\lambda| \leq r\}} e^{\lambda t+i \tilde{\xi} \cdot \tilde{x}}\left(L_{\tilde{\xi}}-\lambda\right)^{-1} d \lambda d \tilde{\xi}
$$

Proof of bounds on $\mathcal{S}_{1}(t)$. We first prove (2.2) for $\beta=0$. Let $\hat{u}\left(x_{1}, \tilde{\xi}, \lambda\right)$ denote the solution of $\left(L_{\tilde{\xi}}-\lambda\right) \hat{u}=\hat{f}$, where $\hat{f}\left(x_{1}, \tilde{\xi}\right)$ denotes Fourier transform of $f$, and

$$
u(x, t):=\mathcal{S}_{1}(t) f=\frac{1}{(2 \pi i)^{d}} \int_{|\tilde{\xi}| \leq r} \oint_{\Gamma \tilde{\xi} \cap\{|\lambda| \leq r\}} e^{\lambda t+i \tilde{\xi} \cdot \tilde{x}}\left(L_{\tilde{\xi}}-\lambda\right)^{-1} \hat{f}\left(x_{1}, \tilde{\xi}\right) d \lambda d \tilde{\xi} .
$$

Using Parseval's identity, Fubini's theorem, the triangle inequality, and Proposition 2.2, we may estimate

$$
\begin{aligned}
|u|_{L^{2}\left(x_{1}, \tilde{x}\right)}^{2}(t) & =\frac{1}{(2 \pi)^{2 d}} \int_{x_{1}} \int_{|\tilde{\xi}| \leq r}\left|\oint_{\Gamma_{\tilde{\xi}} \cap\{|\lambda| \leq r\}} e^{\lambda t} \hat{u}\left(x_{1}, \tilde{\xi}, \lambda\right) d \lambda\right|^{2} d \tilde{\xi} d x_{1} \\
& \leq\left.\left.\frac{1}{(2 \pi)^{2 d}} \int_{\tilde{\xi}}\left|\oint_{\Gamma_{\tilde{\xi}} \cap\{|\lambda| \leq r\}} e^{\Re e \lambda t}\right| \hat{u}\left(x_{1}, \tilde{\xi}, \lambda\right)\right|_{L^{2}\left(x_{1}\right)} d \lambda\right|^{2} d \tilde{\xi} \\
& \leq C|f|_{L^{1}(x)}^{2} \int_{\tilde{\xi}}\left|\oint_{\Gamma^{\tilde{\xi}} \cap\{|\lambda| \leq r\}} e^{\Re e \lambda t} \rho^{-3 / 2} d \lambda\right|^{2} d \tilde{\xi} .
\end{aligned}
$$

Specifically, parametrizing $\Gamma^{\tilde{\xi}}$ by

$$
\lambda(\tilde{\xi}, k)=i k-\theta_{1}\left(k^{2}+|\tilde{\xi}|^{2}\right), \quad k \in \mathbb{R}
$$


and noting that $|d \lambda / d k|$ is bounded on $\Gamma^{\tilde{\xi}} \cap\{|\lambda| \leq r\}$, we estimate

$$
\begin{aligned}
\int_{\tilde{\xi}}\left|\oint_{\Gamma \tilde{\xi} \cap\{|\lambda| \leq r\}} e^{\Re e \lambda t} \rho^{-3 / 2} d \lambda\right|^{2} d \tilde{\xi} & \leq C \int_{\tilde{\xi}}\left|\int_{\mathbb{R}} e^{-\theta_{1}\left(k^{2}+|\tilde{\xi}|^{2}\right) t} \rho^{-3 / 2} d k\right|^{2} d \tilde{\xi} \\
& \leq\left.\left. C \int_{\tilde{\xi}} e^{-2 \theta_{1}|\tilde{\xi}|^{2} t}|\tilde{\xi}|^{-1-2 \epsilon}\left|\int_{\mathbb{R}} e^{-\theta_{1} k^{2} t}\right| k\right|^{\epsilon-1} d k\right|^{2} d \tilde{\xi} \\
& \leq C t^{-(d-2) / 2},
\end{aligned}
$$

noting that $\int_{\mathbb{R}^{d-1}} e^{-\theta|x|^{2}}|x|^{-\alpha} d x$ is finite, provided $\alpha<d-1$.

Similarly, parametrizing $\Gamma^{\tilde{\xi}}$ as above, we estimate

$$
\begin{aligned}
|u|_{L_{\tilde{x}, x_{1}}^{\infty}}(t) & \leq \frac{1}{(2 \pi)^{d}} \int_{\tilde{\xi}} \oint_{\Gamma_{\tilde{\xi}} \cap\{|\lambda| \leq r\}} e^{\Re e \lambda t}\left|\hat{u}\left(x_{1}, \tilde{\xi}, \lambda\right)\right|_{L^{\infty}\left(x_{1}\right)} d \lambda d \tilde{\xi} \\
& \leq C|f|_{L^{1}(x)} \int_{\tilde{\xi}} \oint_{\Gamma_{\tilde{\xi}} \cap\{|\lambda| \leq r\}} e^{\Re e \lambda t} \rho^{-3 / 2} d \lambda d \tilde{\xi} \\
& \leq \int_{\tilde{\xi}} e^{-\theta_{1}|\tilde{\xi}|^{2} t}|\tilde{\xi}|^{-1 / 2-\epsilon} \int_{\mathbb{R}} e^{-\theta_{1} k^{2} t}|k|^{\epsilon-1} d k d \tilde{\xi} \\
& \leq C t^{-\frac{d-1}{2}+\frac{1}{4}}
\end{aligned}
$$

The $x_{1}$-derivative bounds follow similarly by using the version of the $L^{1} \rightarrow L^{p}$ estimates for $\beta_{1}=1$, noting that in the undercompressive case, both $\beta_{1}=0$ and $\beta_{1}=1$ have the same bounds. The $\tilde{x}$-derivative bounds are straightforward by the fact that $\partial_{\tilde{x}}^{\tilde{\beta}} f=(i \tilde{\xi})^{\tilde{\beta}} \hat{f}$.

2.5. Proof of linearized stability. Applying estimates (2.2) and (2.4) on low- and highfrequency operators $\mathcal{S}_{1}(t)$ and $\mathcal{S}_{2}(t)$ obtained in Proposition 2.1, we obtain

$$
\begin{aligned}
|U(t)|_{L^{2}} & \leq\left|\mathcal{S}_{1}(t) U_{0}\right|_{L^{2}}+\left|\mathcal{S}_{2}(t) U_{0}\right|_{L^{2}} \\
& \leq C(1+t)^{-\frac{d-2}{4}}\left|U_{0}\right|_{L^{1}}+C e^{-\eta t}\left|U_{0}\right|_{L^{2}} \\
& \leq C(1+t)^{-\frac{d-2}{4}}\left|U_{0}\right|_{L^{1} \cap L^{2}}
\end{aligned}
$$

and (together with the Sobolev embedding: $|f|_{L^{\infty}\left(\mathbb{R}^{d}\right)} \leq C|f|_{H^{s}\left(\mathbb{R}^{d}\right)}$ for $s>d / 2$; see, for example, [Z4, Lemma 1.4])

$$
\begin{aligned}
|U(t)|_{L^{\infty}} & \leq\left|\mathcal{S}_{1}(t) U_{0}\right|_{L^{\infty}}+\left|\mathcal{S}_{2}(t) U_{0}\right|_{L^{\infty}} \\
& \leq C(1+t)^{-\frac{d-1}{2}+\frac{1}{4}}\left|U_{0}\right|_{L^{1}}+C\left|\mathcal{S}_{2}(t) U_{0}\right|_{H^{[(d-1) / 2]+2}} \\
& \leq C(1+t)^{-\frac{d-1}{2}+\frac{1}{4}}\left|U_{0}\right|_{L^{1}}+C e^{-\eta t}\left|U_{0}\right|_{H^{[(d-1) / 2]+2}} \\
& \leq C(1+t)^{-\frac{d-1}{2}+\frac{1}{4}}\left|U_{0}\right|_{L^{1} \cap H^{[(d-1) / 2]+2} .}
\end{aligned}
$$

These prove the bounds as stated in the theorem for $p=2$ and $p=\infty$. For $2<p<\infty$, we use the interpolation inequality between $L^{2}$ and $L^{\infty}$. 


\section{NONLINEAR STABILITY}

Defining the perturbation variable $U:=\tilde{U}-\bar{U}$, we obtain the nonlinear perturbation equations

$$
U_{t}-L U=\sum_{j} Q^{j}\left(U, U_{x}\right)_{x_{j}}
$$

where

$$
\begin{aligned}
Q^{j}\left(U, U_{x}\right) & =\mathcal{O}\left(|U|\left|U_{x}\right|+|U|^{2}\right) \\
Q^{j}\left(U, U_{x}\right)_{x_{j}} & =\mathcal{O}\left(|U|\left|U_{x}\right|+|U|\left|U_{x x}\right|+\left|U_{x}\right|^{2}\right)
\end{aligned}
$$

so long as $|U|$ remains bounded.

Proof of Theorem 1.8. We prove the theorem for the Lax or overcompressive case. The undercompressive case follows very similarly. Define

$$
\zeta(t):=\sup _{0 \leq s \leq t}\left(|U(s)|_{L^{2}}(1+s)^{\frac{d-2}{4}}+|U(s)|_{L^{\infty}}(1+s)^{\frac{d-1}{2}-\frac{1}{4}}\right) .
$$

We shall prove here that for all $t \geq 0$ for which a solution exists with $\zeta(t)$ uniformly bounded by some fixed, sufficiently small constant, there holds

$$
\zeta(t) \leq C\left(\left|U_{0}\right|_{L^{1} \cap H^{s}}+\zeta(t)^{2}\right) .
$$

This bound together with continuity of $\zeta(t)$ implies that

$$
\zeta(t) \leq 2 C\left|U_{0}\right|_{L^{1} \cap H^{s}}
$$

for $t \geq 0$, provided that $\left|U_{0}\right|_{L^{1} \cap H^{s}}<1 / 4 C^{2}$. This would complete the proof of the bounds as claimed in the theorem, and thus give the main theorem.

By standard short-time theory/local well-posedness in $H^{s}$, and the standard principle of continuation, there exists a solution $U \in H^{s}$ on the open time-interval for which $|U|_{H^{s}}$ remains bounded, and on this interval $\zeta(t)$ is well-defined and continuous. Now, let $[0, T)$ be the maximal interval on which $|U|_{H^{s}}$ remains strictly bounded by some fixed, sufficiently small constant $\delta>0$. By an auxiliary energy estimate in [Z3, Proposition 5.9] and the Sobolev embeding inequality $|U|_{W^{2, \infty}} \leq C|U|_{H^{s}}$ (again, see for example, [Z4, Lemma 1.4]), we have

$$
\begin{aligned}
|U(t)|_{H^{s}}^{2} & \leq C e^{-\theta t}\left|U_{0}\right|_{H^{s}}^{2}+C \int_{0}^{t} e^{-\theta(t-\tau)}|U(\tau)|_{L^{2}}^{2} d \tau \\
& \leq C\left(\left|U_{0}\right|_{H^{s}}^{2}+\zeta(t)^{2}\right)(1+t)^{-(d-2) / 2} .
\end{aligned}
$$

and so the solution continues so long as $\zeta$ remains small, with bound (3.5), yielding existence and the claimed bounds.

Thus, it remains to prove the claim (3.4). By Duhamel formula

$$
U(x, t)=\mathcal{S}(t) U_{0}+\int_{0}^{t} \mathcal{S}(t-s) \sum_{j} \partial_{x_{j}} Q^{j}\left(U, U_{x}\right) d s,
$$


where $U(x, 0)=U_{0}(x)$, we obtain

$$
|U(t)|_{L^{2}} \leq\left|\mathcal{S}(t) U_{0}\right|_{L^{2}}+\int_{0}^{t}\left|\mathcal{S}_{1}(t-s) \partial_{x_{j}} Q^{j}(s)\right|_{L^{2}} d s+\int_{0}^{t}\left|\mathcal{S}_{2}(t-s) \partial_{x_{j}} Q^{j}(s)\right|_{L^{2}} d s
$$

where $\left|\mathcal{S}(t) U_{0}\right|_{L^{2}} \leq C(1+t)^{-\frac{d-2}{4}}\left|U_{0}\right|_{L^{1} \cap L^{2}}$ as in the proof of linearized stability,

$$
\begin{aligned}
\int_{0}^{t}\left|\mathcal{S}_{1}(t-s) \partial_{x_{j}} Q^{j}(s)\right|_{L^{2}} d s & \leq C \int_{0}^{t}(1+t-s)^{-\frac{d-2}{4}-\frac{1}{2}}\left|Q^{j}(s)\right|_{L^{1}} d s \\
& \leq C \int_{0}^{t}(1+t-s)^{-\frac{d-2}{4}-\frac{1}{2}}|U|_{H^{1}}^{2} d s \\
& \leq C\left(\left|U_{0}\right|_{H^{s}}^{2}+\zeta(t)^{2}\right) \int_{0}^{t}(1+t-s)^{-\frac{d-2}{4}-\frac{1}{2}}(1+s)^{-\frac{d-2}{2}} \\
& \leq C(1+t)^{-\frac{d-2}{4}}\left(\left|U_{0}\right|_{H^{s}}^{2}+\zeta(t)^{2}\right)
\end{aligned}
$$

and

$$
\begin{aligned}
\int_{0}^{t}\left|\mathcal{S}_{2}(t-s) \partial_{x_{j}} Q^{j}(s)\right|_{L^{2}} d s & \leq \int_{0}^{t} e^{-\theta(t-s)}\left|\partial_{x_{j}} Q^{j}(s)\right|_{L^{2}} d s \\
& \leq C \int_{0}^{t} e^{-\theta(t-s)}|U|_{H^{s}}^{2} d s \\
& \leq C\left(\left|U_{0}\right|_{H^{s}}^{2}+\zeta(t)^{2}\right) \int_{0}^{t} e^{-\theta(t-s)}(1+s)^{-\frac{d-2}{2}} d s \\
& \leq C(1+t)^{-\frac{d-2}{2}}\left(\left|U_{0}\right|_{H^{s}}^{2}+\zeta(t)^{2}\right) .
\end{aligned}
$$

Thus, dividing by $(1+t)^{-\frac{d-2}{4}}$, we obtain

$$
|U(t)|_{L^{2}}(1+t)^{\frac{d-2}{4}} \leq C\left(\left|U_{0}\right|_{L^{1} \cap H^{s}}+\zeta(t)^{2}\right) .
$$

Similarly, we estimate the $L^{\infty}$ norm of $U$. By Duhamel's formula (3.7), we obtain

$$
\begin{aligned}
|U(t)|_{L^{\infty}} \leq & \left|\mathcal{S}(t) U_{0}\right|_{L^{\infty}}+\int_{0}^{t}\left|\mathcal{S}_{1}(t-s) \partial_{x_{j}} Q^{j}(s)\right|_{L^{\infty}} d s \\
& +\int_{0}^{t}\left|\mathcal{S}_{2}(t-s) \partial_{x_{j}} Q^{j}(s)\right|_{L^{\infty}} d s
\end{aligned}
$$


where $\left|\mathcal{S}(t) U_{0}\right|_{L^{\infty}} \leq C(1+t)^{-\frac{d-1}{2}+\frac{1}{4}}\left|U_{0}\right|_{L^{1} \cap H^{[(d-1) / 2]+2}}$,

$$
\begin{aligned}
\int_{0}^{t} \mid \mathcal{S}_{1}(t-s) & \left.\partial_{x_{j}} Q^{j}(s)\right|_{L^{\infty}} d s \\
& \leq C \int_{0}^{t}(1+t-s)^{-\frac{d-1}{2}+\frac{1}{4}-\frac{1}{2}}\left|Q^{j}(s)\right|_{L^{1}} d s \\
& \leq C \int_{0}^{t}(1+t-s)^{-\frac{d-1}{2}+\frac{1}{4}-\frac{1}{2}}|U|_{H^{1}}^{2} \\
& \leq C\left(\left|U_{0}\right|_{H^{s}}^{2}+\zeta(t)^{2}\right) \int_{0}^{t}(1+t-s)^{-\frac{d-1}{2}+\frac{1}{4}-\frac{1}{2}}(1+s)^{-\frac{d-2}{2}} \\
& \leq C(1+t)^{-\frac{d-1}{2}+\frac{1}{4}}\left(\left|U_{0}\right|_{H^{s}}^{2}+\zeta(t)^{2}\right)
\end{aligned}
$$

and (by the Moser inequality; see, for example, inequality (1.22), [Z4]),

$$
\begin{aligned}
\int_{0}^{t} \mid \mathcal{S}_{2}(t-s) & \left.\partial_{x_{j}} Q^{j}(s)\right|_{L^{\infty}} d s \\
& \leq \int_{0}^{t}\left|\mathcal{S}_{2}(t-s) \partial_{x_{j}} Q^{j}(s)\right|_{H^{[(d-1) / 2]+2}} d s \\
& \leq \int_{0}^{t} e^{-\theta(t-s)}\left|\partial_{x} Q^{j}(s)\right|_{H^{[(d-1) / 2]+2}} d s \\
& \leq C \int_{0}^{t} e^{-\theta(t-s)}|U|_{L^{\infty}}|U|_{H^{[(d-1) / 2]+4}} d s \\
& \leq C\left(\left|U_{0}\right|_{H^{s}}^{2}+\zeta(t)^{2}\right) \int_{0}^{t} e^{-\theta(t-s)}(1+s)^{-\frac{d-1}{2}+\frac{1}{4}}(1+s)^{-\frac{d-2}{4}} d s \\
& \leq C(1+t)^{-\frac{d-1}{2}+\frac{1}{4}}\left(\left|U_{0}\right|_{H^{s}}^{2}+\zeta(t)^{2}\right) .
\end{aligned}
$$

Therefore we have obtained

$$
|U(t)|_{L^{\infty}}(1+t)^{\frac{d-1}{2}-\frac{1}{4}} \leq C\left(\left|U_{0}\right|_{L^{1} \cap H^{s}}+\zeta(t)^{2}\right)
$$

and thus completed the proof of claim (3.4), and the theorem.

\section{TWo-Dimensional CASE OR CASES With (H5)}

In this section, we give a proof of Theorem 1.10, Again, notice that the only assumption we make here that differs from those in [Z3] is the relaxed Hypothesis (H4'), treating the additional case of totally nonglancing characteristic roots, which is only involved in lowfrequency estimates. That is to say, we only need to establish the $L^{1} \rightarrow L^{p}$ bounds in low-frequency regimes for this new case. We give the proof of these bounds by modifying the proof in [GMWZ1, Section 12] and thus will not cite the estimate (2.16) in this section; in fact, the proof is completely independent of previous sections. In addition, our proof is somewhat more direct and simpler than those in [GMWZ1, Section 12] by not bypassing to the dual problem. 
Proposition 4.1 (Low-frequency bounds; [Z3], Corollary 5.11). Under the hypotheses of Theorem 1.10, for $\lambda \in \Gamma^{\tilde{\xi}}$ (see (2.17)) and $\rho:=|(\tilde{\xi}, \lambda)|, \theta_{1}$ sufficiently small, there holds the resolvent bound

$$
\left|\left(L_{\tilde{\xi}}-\lambda\right)^{-1} \partial_{x_{1}}^{\beta} f\right|_{L^{p}\left(x_{1}\right)} \leq C \gamma_{2} \rho^{\beta-1}|f|_{L^{1}\left(x_{1}\right)},
$$

for all $2 \leq p \leq \infty, \beta=0,1$, and $\gamma_{2}$ is the diagonalization error (see [Z3], (5.40)) defined as

$$
\gamma_{2}:=1+\sum_{j, \pm}\left[\rho^{-1}\left|\Im m \lambda-\eta_{j}^{ \pm}(\tilde{\xi})\right|+\rho\right]^{1 / s_{j}-1}
$$

with $\eta_{j}^{ \pm}, s_{j}$ as in (H5).

We again perform the standard procedure (see Section 2.2) of writing the linearized equations in form of the first order eigenvalue equations (2.12):

$$
\partial_{x_{1}}\left(\begin{array}{c}
U_{H} \\
U_{P}
\end{array}\right)=\left(\begin{array}{cc}
H & 0 \\
0 & P
\end{array}\right)\left(\begin{array}{l}
U_{H} \\
U_{P}
\end{array}\right)+\left(\begin{array}{c}
F_{H} \\
F_{P}
\end{array}\right), \quad \bar{\Gamma} U=0 .
$$

Locally, in a neighborhood of a base point $X_{0}:=(\underline{\zeta}, 0)$ with $\zeta=(\tau, \gamma, \tilde{\xi})$ and $\lambda=\gamma+i \tau$, we further use the Assumption (H4') to write $H$ in block-diagonal structure (see GMWZ1, Proposition 6.1]) with appearance of a new mode, totally nonglancing, and decompose the resolvent solution $U$ into

$$
U=U_{P}+U_{H_{e}}+U_{H_{h}}+U_{H_{g}}+U_{H_{t}},
$$

corresponding to parabolic, elliptic, hyperbolic, glancing, or totally nonglancing blocks. We further write

$$
U_{i}=U_{i+}+U_{i-}
$$

for $i=P, H_{e}, H_{h}, H_{g}, H_{t}$, where $U_{i \pm}$ are defined as the projections of $U_{i}$ onto the growing (resp. decaying) eigenspaces of $\mathcal{G}_{+}$in (2.10) with respect to the corresponding blocks.

These first four blocks have been treated in [Z3, Corollary 5.11] or [GMWZ1, Corollary 12.2] for which the totally nonglancing modes are absent. For sake of completeness, we treat these modes again here in a slightly different analysis, modifying those of [GMWZ1, Section 12]. In fact, since each mode interacts with the other via the Evans condition (D) or, more precisely, the boundary estimate (A.28), we cannot obtain (4.1) for each mode separately.

We shall use the following simple lemma.

Lemma 4.2. Let $U$ be a solution of $\partial_{z} U=Q U+F$ with $U(+\infty)=0$. Assume that there is a positive [resp., negative] symmetric matrix $S$ such that

$$
\Re S Q:=\frac{1}{2}\left(S Q+Q^{*} S^{*}\right) \geq \theta I d
$$

for some $\theta>0$, and $S \geq I d$ [resp., $-S \geq I d]$. Then there holds

$$
\begin{aligned}
&|U|_{L^{\infty}}^{2}+\theta|U|_{L^{2}}^{2} \lesssim|F|_{L^{1}}^{2} \\
&\text { [resp., } \left.|U|_{L^{\infty}}^{2}+\theta|U|_{L^{2}}^{2} \lesssim|U(0)|^{2}+|F|_{L^{1}}^{2}\right] .
\end{aligned}
$$


Proof. Taking the real part of the inner product of the equation of $U$ against $S U$ and integrating the result over $\left[x_{1}, \infty\right]$ for the first case [resp., $\left[0, x_{1}\right]$ for the second case], we easily obtain the lemma.

Proof of Proposition 4.1. As in [GMWZ1, Section 12.2], the first step is to put blocks into a diagonal form; indeed, parabolic blocks are already diagonal as in (2.11); hyperbolic blocks are $1 \times 1$ blocks with real part vanishing at the base point $X_{0}$, but with real part $>0$ (resp. $<0$ ) when $\rho>0$ in polar coordinates (thus, vanishing at order $\rho^{2}$ in original coordinates); elliptic blocks are those $Q_{k}$ with $\Re e Q_{k}$ positive or negative definite at the base point (thus, vanishing at order $\rho$ ); and finally glancing blocks are of size larger than $1 \times 1$ whose components are purely imaginary at the base point. We recall the following lemma in [GMWZ1, diagonalizing these glancing blocks.

Lemma 4.3 (Lemma 12.1, GMWZ1]). Diagonalize the glancing blocks $Q_{k}$ by the transformation $T_{H_{g}}$, where $T_{H_{g}}$ may be chosen so that

$$
\left|T_{H_{g}}\right| \leq C, \quad\left|T_{H_{g}}^{-1}\right| \leq C \gamma_{2}, \quad\left|T_{\left.H_{g}\right|_{H_{g-}}}^{-1}\right| \leq C \gamma_{1}
$$

where $\gamma_{2}$ is defined as in (4.2) and $\gamma_{1}$ is defined as

$$
\gamma_{1}:=\max _{k}\left[\rho^{-1}\left|\Im m \lambda-\eta_{k}^{ \pm}(\tilde{\xi})\right|+\rho\right]^{\left(1-\left[\left(\nu_{k}+1\right) / 2\right]\right) / \nu_{k}}
$$

and $T_{\left.H_{g}\right|_{H_{g-}}}^{-1}$ denotes the restriction of $T_{H_{g}}^{-1}$ to subspace $H_{g-}$.

In addition, after a further transformation if necessary,

$$
Q_{k}^{\prime}:=T_{H_{g}}^{-1} Q_{k} T_{H_{g}}=\operatorname{diag}\left(\alpha_{k, 1}, \cdots, \alpha_{k, l}, \alpha_{k, l+1}, \cdots, \alpha_{k, \nu_{k}}\right)
$$

with

$$
\begin{aligned}
-\gamma_{1}^{-2} \Re e \alpha_{k, j} \geq C \rho^{2}, & j=1, \ldots, l, \\
\gamma_{1}^{-2} \Re e \alpha_{k, j} \geq C \rho^{2}, & j=l+1, \ldots, \nu_{k} .
\end{aligned}
$$

Remark 4.4. $\gamma_{1}, \gamma_{2}$ are identical to $\alpha, \beta$ in [GMWZ1], respectively, and (4.10) was calculated in [GMWZ1, equation (12.40)].

We now can work in diagonalized coordinates:

$$
U^{\prime}:=T_{H_{g}}^{-1} U
$$

where $T_{H_{g}}$ are obtained as in Lemma 4.3 for glancing blocks and identity matrices for the other blocks. In these coordinates, since blocks are diagonal and growing/decaying subspaces (at least for the first four modes) are separated, we apply Lemma 4.2 for each block with $S= \pm I d$, yielding

$$
\begin{aligned}
& \left|U_{i+}^{\prime}\right|_{L^{\infty}}^{2}+\theta_{i}\left|U_{i+}^{\prime}\right|_{L^{2}}^{2} \lesssim\left|F_{i}^{\prime}\right|_{L^{1}}^{2}, \\
& \left|U_{i-}^{\prime}\right|_{L^{\infty}}^{2}+\theta_{i}\left|U_{i-}^{\prime}\right|_{L^{2}}^{2} \lesssim\left|U_{i-}^{\prime}(0)\right|^{2}+\left|F_{i-}^{\prime}\right|_{L^{1}}^{2},
\end{aligned}
$$

where $\theta_{i}=1, \rho, \rho^{2}, \min _{j}\left|\Re e \alpha_{k, j}\right|$ for $i=P, H_{e}, H_{h}, H_{g}$.

For the totally nonglancing blocks $Q_{t}^{k}$, as constructed in [GMWZ6, Lemma 5.3, there exist symmetrizers $S^{k}$ that are definite positive [resp., negative] when the mode is totally 
incoming [resp., outgoing]. Denote $U_{H_{t+}}^{\prime}$ [resp., $U_{H_{t-}}^{\prime}$ ] associated with totally incoming [resp. outgoing] modes. Then by applying Lemma 4.2 with $\theta=\rho^{2}$, we obtain

$$
\begin{aligned}
\left|U_{H_{t+}}^{\prime}\right|_{L^{\infty}}^{2}+\rho^{2}\left|U_{H_{t+}}^{\prime}\right|_{L^{2}}^{2} \lesssim\left|F_{H_{t+}}^{\prime}\right|_{L^{1}}^{2} \\
\left|U_{H_{t-}}^{\prime}\right|_{L^{\infty}}^{2}+\rho^{2}\left|U_{H_{t-}}^{\prime}\right|_{L^{2}}^{2} \lesssim\left|U_{H_{t-}}^{\prime}(0)\right|^{2}+\left|F_{H_{t-}}^{\prime}\right|_{L^{1}}^{2} .
\end{aligned}
$$

To finish the proof, we only need to deal with the boundary terms $\left|U_{i-}^{\prime}(0)\right|^{2}$ and $\left|U_{H_{t-}}(0)\right|^{2}$ in (4.11), (4.12). We could use a more detailed version of the $L^{2}$ stability estimate (2.16), corresponding to each diagonal blocks (see [GMWZ6]), yielding bounds on these boundary terms. However, let us now follow the boundary treatment presented in GMWZ1, Section 12.3] instead, being rather independent of (2.16).

The diagonalized boundary condition is $\Gamma^{\prime}:=\Gamma T_{H_{g}}$. By computing, we observe that

$$
\left|\Gamma^{\prime} U_{H_{g-}}^{\prime}\right|=\left|\Gamma U_{H_{g-}}\right| \geq C^{-1}\left|U_{H_{g-}}\right| \geq \frac{C^{-1}\left|U_{H_{g-}}^{\prime}\right|}{\left|T_{\left.H_{g}\right|_{H_{g-}}}^{-1}\right|} \geq C^{-1} \gamma_{1}^{-1}\left|U_{H_{g-}}^{\prime}\right| .
$$

Thus, together with (A.28)

$$
\left|\Gamma^{\prime} U_{-}^{\prime}\right|=\left|\Gamma U_{-}\right| \geq C^{-1}\left[\left|U_{H_{e-}}^{\prime}\right|+\left|U_{H_{h-}}^{\prime}\right|+\left|U_{H_{t-}}^{\prime}\right|+\gamma_{1}^{-1}\left|U_{H_{g-}}^{\prime}\right|+\rho\left|U_{P_{-}}^{\prime}\right|\right] .
$$

Meanwhile, we have at $x_{1}=0$

$$
\left|\Gamma^{\prime} U_{-}^{\prime}\right| \leq\left|\Gamma^{\prime} U^{\prime}\right|+\left|\Gamma^{\prime} U_{+}^{\prime}\right| \lesssim\left|U_{+}^{\prime}(0)\right| \leq\left|U_{+}^{\prime}\right|_{L^{\infty}}
$$

Now, multiplying the first equations in (4.11), (4.12) by a sufficiently large constant $k$ and the second equations by $1, \gamma_{1}^{-2}$, or $\rho^{2}$, corresponding to each block with its boundary degeneracy of order in (4.13), and adding up the results, we easily obtain

$$
\begin{aligned}
& \left|U_{P+}^{\prime}\right|_{L^{\infty}}^{2}+\left|U_{P+}^{\prime}\right|_{L^{2}}^{2}+\left|U_{H+}^{\prime}\right|_{L^{\infty}}^{2}+\rho^{2}\left|U_{H+}^{\prime}\right|_{L^{2}}^{2}+ \\
& \rho^{2}\left|U_{P-}^{\prime}\right|_{L^{\infty}}^{2}+\rho^{2}\left|U_{P-}^{\prime}\right|_{L^{2}}^{2}+\gamma_{1}^{-2}\left|U_{H-}^{\prime}\right|_{L^{\infty}}^{2}+\rho^{2}\left|U_{H-}^{\prime}\right|_{L^{2}}^{2} \lesssim\left|F^{\prime}\right|_{L^{1}}^{2},
\end{aligned}
$$

(noting that $\rho \leq 1, \rho \leq \gamma_{1}^{-1} \leq 1$, and $\gamma_{1}^{-2} \min _{j}\left|\Re e \alpha_{k, j}\right| \gtrsim \rho^{2}$ by (4.10)). This yields

$$
\rho^{2}\left|U^{\prime}\right|_{L^{\infty}}^{2}+\rho^{2}\left|U^{\prime}\right|_{L^{2}}^{2} \lesssim\left|F^{\prime}\right|_{L^{1}}^{2}
$$

or equivalently,

$$
\left|U^{\prime}\right|_{L^{p}} \lesssim \rho^{-1}\left|F^{\prime}\right|_{L^{1}}, \quad \forall p \geq 2
$$

Thus, by recalling that $U=T_{H_{g}} U^{\prime}$ and $F^{\prime}=T_{H_{g}}^{-1} F$, (4.17) and (4.7) immediately yield the proposition for $\beta=0$. For $\beta=1$, we can follow the Kreiss-Kreiss trick as presented in the proof of Proposition 2.2, thus completing the proof of Proposition 4.1.

Proof of Theorem 1.10. Proposition 4.1] is the Corollary 5.1 in [Z3] with an extension to the totally nonglancing cases. Thus, we can now follow word by word the proof in [Z3], yielding the theorem.

Remark 4.5. We have seen in the above argument that the existence of positive/negative Kreiss' symmetrizers with an appropriate constant $\theta$ (in Lemma 4.2) is sufficient to obtain the result. Though, proving the existence of such symmetrizers is a highly-technical task in general for variable multiplicity blocks. See [GMWZ5, GMWZ6]. 


\section{Appendix A. Evans FunCtion For the Doubled BOUNDARY PROBlem}

For sake of completeness, we recall here the proof of Lemma 7.1, GMWZ1 and its straightforward extension to the case of over- and under-compressive shocks.

Consider the $2 N \times 2 N$ doubled boundary problem (2.7) (with $N:=n+r$ )

$$
\left\{\begin{array}{l}
U_{x}-G(x, \zeta) U=F, \\
\Gamma U=0 \text { on } x=0,
\end{array}\right.
$$

where $U=\left(U_{+}, U_{-}\right)$and $\Gamma U=U_{+}-U_{-}$, with $U_{+}=\left(U_{1}, \ldots, U_{N}\right), U_{-}=\left(U_{N+1}, \ldots, U_{2 N}\right)$.

Let $\mathcal{E}_{-}(\hat{\zeta}, \rho)$, for $\hat{\gamma}>0$ and $\rho>0$, be the space of boundary values at $x=0$ of decaying solutions to the homogeneous problem

$$
U_{x}-G(x, \zeta) U=0 .
$$

As shown, for example, in [GMWZ6, Theorem 3.7], the space $\mathcal{E}_{-}(\hat{\zeta}, \rho)$ has a continuous extension to a small neighborhood of $\hat{\gamma}=0, \rho \geq 0$. Then the Evans function for (A.1) is defined as the $2 N \times 2 N$ determinant:

$$
\mathbb{D}(\hat{\zeta}, \rho)=\left.\operatorname{det}\left(\operatorname{ker} \Gamma, \mathcal{E}_{-}\right)\right|_{x=0} .
$$

Meanwhile, the Evans function $D_{L}$ for the problem (2.6) is defined as

$$
D_{L}(\hat{\zeta}, \rho)=\left.\operatorname{det}\left(\mathcal{U}_{1}^{R}, \ldots, \mathcal{U}_{k}^{R}, \mathcal{U}_{k+1}^{L}, \ldots, \mathcal{U}_{N}^{L}\right)\right|_{x=0}
$$

which is analytic for $\Re e \lambda>0$ and can be continuously extended to a small neighborhood of $\Re e \lambda=0$ (see, e.g., Lemma 5.24, [Z3]). Now let $\phi_{j}, j=1, \ldots, l$ be the derivative of the profile $\bar{U}^{\delta}$ with respect to $\delta_{j}$, where $l$ is the dimension of the smooth manifold $\left\{\bar{U}^{\delta}(\cdot)\right\}$ defined as in (H3). Thanks to the Evans condition (D), without loss of generality, we can assume that

$$
\mathcal{U}_{j}^{R}(x, \hat{\zeta}, 0)=\mathcal{U}_{N-j+1}^{L}(x, \hat{\zeta}, 0)=\left(\phi_{j}(x), 0\right)
$$

for $j=1, \ldots, l$.

Let $e_{j} \in \mathbb{C}^{N}$ be the unit vectors

$$
e_{j}=\frac{\left(\phi_{j}(0), 0\right)}{\left|\phi_{j}(0)\right|}, \quad j=1, \ldots, l
$$

and extend to an orthonormal basis $e_{1}, \ldots, e_{N}$ of $\mathbb{C}^{N}$. Then the Evans function (A.2) for the doubled boundary value problem can be explicitly defined as

$$
\mathbb{D}(\hat{\zeta}, \rho)=\left.\operatorname{det}\left(\begin{array}{ccccccccc}
e_{1} & \ldots & e_{N} & \mathcal{U}_{1}^{R} & \ldots & \mathcal{U}_{k}^{R} & 0 & \ldots & 0 \\
e_{1} & \ldots & e_{N} & 0 & \ldots & 0 & \mathcal{U}_{k+1}^{L} & \ldots & \mathcal{U}_{N}^{L}
\end{array}\right)\right|_{x=0} .
$$

We also set

$$
\mathcal{E}_{-, \phi}(\hat{\zeta}, \rho)=\left.\operatorname{span}\left\{\left(\begin{array}{c}
\mathcal{U}_{1}^{R} \\
\mathcal{U}_{N}^{L}
\end{array}\right), \ldots,\left(\begin{array}{c}
\mathcal{U}_{l}^{R} \\
\mathcal{U}_{N-l+1}^{L}
\end{array}\right)\right\}\right|_{(0, \hat{\zeta}, \rho)}
$$

For $\epsilon>0$ fixed, denote by $\mathcal{E}_{-, \phi, \epsilon}^{c}(\hat{\zeta}, \rho)$ any complementary subspace in $\mathcal{E}_{-}(\hat{\zeta}, \rho)$ varying continuously with $(\hat{\zeta}, \rho)$ such that

$$
\mathcal{E}_{-}(\hat{\zeta}, \rho)=\mathcal{E}_{-, \phi}(\hat{\zeta}, \rho) \oplus \mathcal{E}_{-, \phi, \epsilon}^{c}(\hat{\zeta}, \rho)
$$

with uniformly bounded projections for $0 \leq \rho \leq \epsilon$. 
Then, we recall the following proposition that was proved for the Lax shock case in GMWZ1, Proposition 7.1.

Proposition A.1. (1) Let $D_{L}(\hat{\zeta}, \rho)$ and $\mathbb{D}(\hat{\zeta}, \rho)$ be the Evans functions defined as above. Then

$$
D_{L}(\hat{\zeta}, \rho)=(-1)^{N} \mathbb{D}(\hat{\zeta}, \rho) .
$$

(2) Under the Evans assumption (D), we have the following.

(a) For any choice of $0<\delta<R$ there is a constant $C_{\delta}, R$ such that when $\delta \leq \rho \leq R$,

$$
|\Gamma u| \geq C_{\delta, R}|u| \quad \text { for } u \in \mathcal{E}_{-}(\hat{\zeta}, \rho) .
$$

(b) There exist positive constants $C_{1}, C_{2}, \delta$ such that

$$
C_{1} \rho|u| \leq|\Gamma u| \leq C_{2} \rho|u| \quad \text { for } u \in \mathcal{E}_{-, \phi}(\hat{\zeta}, \rho)
$$

for $0 \leq \rho \leq \delta$.

(c) There exists $C>0$ such that

$$
|\Gamma u| \geq C|u| \quad \text { for } u \in \mathcal{E}_{-, \phi, \epsilon}^{c}(\hat{\zeta}, \rho)
$$

for $0 \leq \rho \leq \epsilon$.

(d) For any choice of $R>0$ there is a constant $C_{R}$ such that for $0 \leq \rho \leq R$,

$$
|\Gamma u| \geq C_{R} \rho|u| \quad \text { for } u \in \mathcal{E}_{-}(\hat{\zeta}, \rho) .
$$

Proof. We follow word by word the proof for the Lax shock case in GMWZ1, Proposition 7.1. First, by performing the row matrix operation, (1) is clear. (2a) follows by continuity and compactness, and the fact that $\Gamma u$ is nonvanishing for nonzero $u \in \mathcal{E}_{-}(\hat{\zeta}, \rho)$ when $\rho>0$ by Evans function assumption (D).

For the proof of $(2 \mathrm{~b})$, let us denote the matrix in (A.5) by $\mathcal{M}$ and perform column operations to replace the last $l$ columns of $\mathcal{M}$ by $\left(\begin{array}{c}\mathcal{U}_{j}^{R} \\ \mathcal{U}_{N-j+1}^{L}\end{array}\right)$, and call the resulting matrix $\mathcal{M}_{1}$. Now thanks to the normalization (A.4) and the fact that fast modes depend analytically on $\rho$, we have for $j=1, \ldots, l$

$$
\left(\begin{array}{c}
\mathcal{U}_{j}^{R} \\
\mathcal{U}_{N-j+1}^{L}
\end{array}\right)(0, \hat{\zeta}, \rho)=\left(\begin{array}{c}
\left(\phi_{j}(0), 0\right) \\
\left(\phi_{j}(0), 0\right)
\end{array}\right)+\left(\begin{array}{c}
c_{1 j}(\hat{\zeta}) \\
c_{2 j}(\hat{\zeta})
\end{array}\right) \rho+\mathcal{O}\left(\rho^{2}\right)
$$

Thus, the definition of $e_{j}$, linearity of the determinant in the last $l$ columns, and the Evans condition (D) show that $c_{1 j}-c_{2 j}$ are nonzero for all $j$. This together with the definition of $\Gamma U$

$$
\Gamma\left(\begin{array}{l}
U^{R} \\
U^{L}
\end{array}\right)=U^{R}-U^{L}
$$

yields (A.10) at once.

(2c) Let $v_{1}(\hat{\zeta}, \rho), \cdots, v_{N}(\hat{\zeta}, \rho)$ be the last $2 n$ columns of the matrix $\mathcal{M}_{1}$ defined above. These vectors form a basis for $\mathcal{E}_{-}(\hat{\zeta}, \rho)$. Take an arbitrary vector $w \in \mathcal{E}_{-, \phi, \epsilon}^{c}(\hat{\zeta}, \rho)$. Then

$$
w=\sum_{j=1}^{N} c_{j, \epsilon}(\hat{\zeta}, \rho) v_{j}(\hat{\zeta}, \rho),
$$


where $c_{j, \epsilon}(\hat{\zeta}, \rho)$ depend continuously on $(\hat{\zeta}, \rho)$.

Set $c_{\epsilon}^{\prime}=\left(c_{1, \epsilon}, \cdots, c_{N-l, \epsilon}\right)$ and $c_{\epsilon}^{\prime \prime}=\left(c_{N-l+1, \epsilon}, \cdots, c_{N, \epsilon}\right)$. The condition that the projections in (A.7) are uniformly bounded implies that there is an $\epsilon_{0}>0$ such that

$$
\left|c_{\epsilon}^{\prime}(\hat{\zeta}, \rho)\right| \geq \epsilon_{0}\left|c_{\epsilon}^{\prime \prime}(\hat{\zeta}, \rho)\right|
$$

for $0 \leq \rho \leq \epsilon$.

In view of (D), we just need to show that $\Gamma w$ is nonvanishing at $\rho=0$ for $w$ as in (A.14) and (A.15) with $\left|\left(c_{\epsilon}^{\prime}, c_{\epsilon}^{\prime \prime}\right)\right|=1$, since (A.11) then follows by continuity and compactness. Suppose $\Gamma w=0$ at $(\hat{\zeta}, 0)$ for some such $w$. Because of (A.15) some $c_{j, \epsilon}$ with $j \leq N-l$, say, $c_{1, \epsilon}$ satisfies

$$
\left|c_{1, \epsilon}\right| \geq c_{0}
$$

for $\rho$ near 0 , and for some $c_{0}>0$. Since $\Gamma w=0$ at $\rho=0$ and $w(\hat{\zeta}, \rho)$ is continuous, we have

$$
w(\hat{\zeta}, \rho)=\left(\begin{array}{c}
a(\hat{\zeta}) \\
a(\hat{\zeta})
\end{array}\right)+\mathcal{O}(\rho) .
$$

Write $v_{j}=\left(v_{j+}, v_{j-}\right)$, use column operations to replace $v_{1}$ in $\mathcal{M}_{1}$ by $w$, and call the resulting matrix $\mathcal{M}_{2}$. Then $\mathcal{M}_{2}=$

$$
\left(\begin{array}{llllllllll}
e_{1} & \ldots & e_{N} & a(\hat{\zeta})+\mathcal{O}(\rho) & v_{2+} & \ldots & v_{N-l,+} & \left(\phi_{1}(0), 0\right)+\mathcal{O}(\rho) & \ldots & \left(\phi_{l}(0), 0\right)+\mathcal{O}(\rho) \\
e_{1} & \ldots & e_{N} & a(\hat{\zeta})+\mathcal{O}(\rho) & v_{2-} & \ldots & v_{N-l,-} & \left(\phi_{1}(0), 0\right)+\mathcal{O}(\rho) & \ldots & \left(\phi_{l}(0), 0\right)+\mathcal{O}(\rho)
\end{array}\right) .
$$

(A.17) implies that $\left|\operatorname{det} \mathcal{M}_{2}(\hat{\zeta}, \rho)\right| \geq C\left|\operatorname{det} \mathcal{M}_{1}(\hat{\zeta}, \rho)\right|$ for some $C>0$ uniformly near $(\hat{\zeta}, 0)$. But

$$
\operatorname{det} \mathcal{M}_{2}(\hat{\zeta}, \rho)=\mathcal{O}(\rho)^{l} \mathcal{O}(\rho) \quad \text { as } \quad \rho \rightarrow 0 .
$$

This contradicts the assumed vanishing of $\operatorname{det} \mathcal{M}=\operatorname{det} \mathcal{M}_{1}$ to exactly $l^{\text {th }}$ order at $\rho=0$.

$(2 \mathrm{~d})$ For any fixed $(\hat{\zeta}, \rho)$, let $u^{*}=\left(\begin{array}{l}u_{+}(\hat{\zeta}, \rho) \\ u_{-}(\hat{\zeta}, \rho)\end{array}\right) \in \mathcal{E}_{-}(\hat{\zeta}, \rho)$ be an element where the minimum

$$
\min _{|u|=1, u \in \mathcal{E}_{-}(\hat{\zeta}, \rho)}|\Gamma u|
$$

is attained. Write $u^{*}=\sum_{j=1}^{N} c_{j, \epsilon}^{*}(\hat{\zeta}, \rho) v_{j}(\hat{\zeta}, \rho)$ and define $c_{j, \epsilon}^{*^{\prime}}, c_{j, \epsilon}^{*^{\prime \prime}}$ in the same way as above. Then, again, the uniform boundedness of the projections in (A.7) implies that there is an $\epsilon_{0}>0$ such that either

$$
\text { (A.18) } \quad\left|c *^{\prime}{ }_{\epsilon}(\hat{\zeta}, \rho)\right| \geq \epsilon_{0}\left|c_{\epsilon}^{*^{\prime \prime}}(\hat{\zeta}, \rho)\right|
$$

or

$$
\left|c *^{\prime \prime}{ }_{\epsilon}(\hat{\zeta}, \rho)\right| \geq \epsilon_{0}\left|c_{\epsilon}^{*^{\prime}}(\hat{\zeta}, \rho)\right|
$$

for $0 \leq \rho \leq \epsilon$. Correspondingly, these imply that, without loss of generality, there holds either

$$
\left|c_{1, \epsilon}^{*}\right| \geq c_{0}
$$

or

$$
\left|c_{N, \epsilon}^{*}\right| \geq c_{0}
$$


for $\rho$ near 0 , and for some $c_{0}>0$.

In the case that A.20 holds, as above, we perform column operations to replace $v_{1}$ in $\mathcal{M}_{1}$ by $u^{*}$, and call the result $\mathcal{M}_{3}$. Then $\mathcal{M}_{3}=$

$$
\left(\begin{array}{llllllllll}
e_{1} & \ldots & e_{N} & u_{+} & v_{2+} & \ldots & v_{N-l,+} & \left(\phi_{1}(0), 0\right)+\mathcal{O}(\rho) & \ldots & \left(\phi_{l}(0), 0\right)+\mathcal{O}(\rho) \\
e_{1} & \ldots & e_{N} & u_{-} & v_{2-} & \ldots & v_{N-l,-} & \left(\phi_{1}(0), 0\right)+\mathcal{O}(\rho) & \ldots & \left(\phi_{l}(0), 0\right)+\mathcal{O}(\rho)
\end{array}\right) .
$$

Next perform column operations to replace the column $u^{*}=\left(\begin{array}{l}u_{+} \\ u_{-}\end{array}\right)$by

$$
\left(\begin{array}{c}
u_{+}-u_{-} \\
0
\end{array}\right)=\left(\begin{array}{c}
\Gamma u^{*} \\
0
\end{array}\right) .
$$

Thus, by direct calculations and (A.20),

$$
\left|\operatorname{det} \mathcal{M}_{3}\right|=\left|\Gamma u^{*}\right| \mathcal{O}(\rho)^{l} \geq C\left|\operatorname{det} \mathcal{M}_{1}\right|=C|\operatorname{det} \mathcal{M}| \geq C \rho^{l},
$$

which gives $\left|\Gamma u^{*}\right| \geq C$.

Similarly, in the case that A.20 holds, replacing $v_{N}$ in $\mathcal{M}_{1}$ by $u^{*}$, denoting the resulting matrix by $\mathcal{M}_{4}$, and performing column operations as above, we then obtain

$$
\left|\operatorname{det} \mathcal{M}_{4}\right|=\left|u_{+}(\hat{\zeta})-u_{-}(\hat{\zeta}, \rho)\right| \mathcal{O}(\rho)^{l-1}=\left|\Gamma u^{*}\right| \mathcal{O}(\rho)^{l-1} \text {. }
$$

This together with $\left|\operatorname{det} \mathcal{M}_{4}\right| \geq C\left|\operatorname{det} \mathcal{M}_{1}\right|=\mathcal{O}\left(\rho^{l}\right)$ by (A.21) yields $\left|\Gamma u^{*}\right| \geq C \rho$.

Thus, altogether we obtain $|\Gamma u| \geq C \rho|u|$, for $u \in \mathcal{E}_{-}(\hat{\zeta}, \rho)$ with $|u|=1$, uniformly in $\rho$ near 0 . Together with $(2 \mathrm{a})$, this proves $(2 \mathrm{~d})$.

Now let $\mathcal{T}$ be the MZ conjugation such that (A.1) leads to the following constantcoefficient system

$$
\left\{\begin{array}{l}
U_{x}-G(\infty, \zeta) U=F \\
\Gamma_{1} U=0 \text { on } x=0
\end{array}\right.
$$

where $\Gamma_{1}=\Gamma \mathcal{T}$ and $G$ has the block form as in (2.10), (2.11):

$$
G(\infty, \zeta)=\left(\begin{array}{ccc}
P_{+}(\zeta) & 0 & 0 \\
0 & P_{-}(\zeta) & 0 \\
0 & 0 & H(\hat{\zeta}, \rho)
\end{array}\right)
$$

Thus, we can decompose $U \in \mathbb{C}^{2 N}$ as follows

$$
U=U_{P_{+}}+U_{P_{-}}+U_{H_{+}}+U_{H_{-}},
$$

and set

$$
U_{-}=U_{P_{-}}+U_{H_{-}} \in \mathcal{E}_{-}(\hat{\zeta}, \rho) .
$$

Define the $l$-dimensional subspace $E_{P_{1-}}$ of $E_{P_{-}}$by

$$
\mathcal{E}_{-, \phi}=\mathcal{T} E_{P_{1-}},
$$

where $\mathcal{E}_{-, \phi}$ is defined as in (A.6), and for $\epsilon>0$ fixed, chose a smoothly varying complementary subspace $E_{P_{2-, \epsilon}}$ such that

$$
\begin{aligned}
& E_{P_{-}}=E_{P_{1-}}(\hat{\zeta}, \rho) \oplus E_{P_{2-, \epsilon}}(\hat{\zeta}, \rho), \\
& U_{P_{-}}=U_{P_{1-}}+U_{P_{2-, \epsilon}}
\end{aligned}
$$


with uniformly bounded projections for $0 \leq \rho \leq \epsilon$. Take

$$
\mathcal{E}_{-, \phi, \epsilon}^{c}=\mathcal{T}\left(E_{P_{2-, \epsilon}} \oplus E_{H_{-}}\right) .
$$

$\mathcal{E}_{-, \phi, \epsilon}^{c}$ is then a choice that works in (A.7).

Then the following is an immediate consequence of Proposition A.1.

Corollary A.2. There exist positive constants $C_{1}, C_{2}$ and $\delta_{0}$ such that for $0 \leq \rho \leq \delta_{0}$

(a) $\quad C_{1} \rho\left|U_{P_{1-}}\right| \leq\left|\Gamma_{1} U_{P_{1-}}\right| \leq C_{2} \rho\left|U_{P_{1-}}\right|$,

(b) $\quad\left|\Gamma_{1}\left(U_{H_{-}}+U_{P_{2-, \epsilon}}\right)\right| \geq C_{1}\left(\left|U_{H_{-}}\right|+\left|U_{P_{2-,}}\right|\right)$,

(c) $\quad\left|\Gamma_{1} U_{-}\right| \geq C_{1} \rho\left|U_{-}\right|$,

where $\Gamma_{1}$ is defined as in (A.22). These estimates hold uniformly near the basepoint $X_{0}=$ $(\underline{\hat{\zeta}}, 0)$.

Thus, we obtain the following lemma which is essential for the construction of degenerate symmetrizers.

Lemma A.3 (Lemma 7.1, GMWZ1]). There exists a constant $\delta>0$ such that for $\rho$ sufficiently small we have

$$
\left|\Gamma U_{-}\right| \geq \delta\left(\left|U_{H_{-}}\right|+\rho\left|U_{P_{-}}\right|\right)
$$

uniformly in a neighborhood of the base point $X_{0}=(\underline{\hat{\zeta}}, 0)$.

Proof. In view of (A.27) (a), (b), we have

$$
\begin{aligned}
\left|\Gamma_{1} U_{-}\right| & =\left|\Gamma_{1} U_{H_{-}}+\Gamma_{1} U_{P_{1-}}+\Gamma_{1} U_{P_{2-}, \epsilon}\right| \\
& \geq C\left(\left|U_{H_{-}}\right|+\left|U_{P_{2-}, \epsilon}\right|\right)-C \rho\left|U_{P_{1-}}\right| .
\end{aligned}
$$

Adding a sufficiently small multiple of this inequality to the inequality (A.27) (c)

$$
\left|\Gamma_{1} U_{-}\right| \geq C \rho\left|U_{-}\right|=C \rho\left(\left|U_{H_{-}}\right|+\left|U_{P_{1-}}\right|+\left|U_{P_{2-}, \epsilon}\right|\right),
$$

we obtain for $\rho$ small

$$
\left|\Gamma_{1} U_{-}\right| \geq \delta\left(\left|U_{-}\right|+\rho\left|U_{P_{1-}}\right|+\left|U_{P_{2-,}}\right|\right)
$$

which implies (A.28).

\section{Appendix B. Auxiliary problem}

In this section we consider the $n \times n$ system on the whole real line $\mathbb{R}$

$$
L_{0} V:=\left(B^{11} V_{x}\right)_{x}-\left(A^{1} V\right)_{x}=f_{x}
$$

where $A^{1}, B^{11}$ are same as in (2.25) . Let us recall $B^{11}=\left(\begin{array}{cc}0 & 0 \\ b_{1}^{11} & b_{2}^{11}\end{array}\right)$. We shall derive an estimate slightly similar to (2.29) by Kreiss-type symmetrizers techniques in the case of Lax and overcompressive shocks. This will be done by modifying the proof in GMWZ1, Section 10.2]; though, our purpose is slightly different and we have to treat the degeneracy of the viscosity matrix $B^{11}$ as comparing to the identity matrix in [GMWZ1. Specifically, we prove the following: 
Lemma B.1. Let $V=\left(V_{1}, V_{2}\right) \in \mathbb{C}^{n-r} \times \mathbb{C}^{r}$ be a solution of (B.1). We prove that there exists a constant $C>0$ such that

$$
\begin{aligned}
& |V|_{L^{p}} \leq C\left(|f|_{L^{1}}+|f|_{L^{\infty}}\right) \\
& \left|V_{x}\right|_{L^{p}} \leq C\left(|f|_{L^{1}}+|f|_{L^{\infty}}+\left|f_{x}\right|_{L^{p}}\right)
\end{aligned}
$$

for any $1 \leq p \leq \infty$.

Proof. We first integrate the equation (B.1), yielding

$$
B^{11} V_{x}-A^{1} V=f .
$$

Consider the double $2 n \times 2 n$ boundary problem on $x \geq 0$ equivalent to (B.3)

$$
\begin{array}{lll}
\mathcal{B} W_{x}-\mathcal{A} W=F & \\
\Gamma W=0 & \text { on } & \{x=0\}
\end{array}
$$

where, defining $\phi_{ \pm}(x)=\phi( \pm x)$ for any function $\phi$ defined on $\mathbb{R}$

$$
\begin{aligned}
& W(x)=\left(\begin{array}{l}
V_{+}(x) \\
V_{-}(x)
\end{array}\right), \\
& \mathcal{A}(x)=\left(\begin{array}{cc}
A_{+}^{1}(x) & 0 \\
0 & -A_{-}^{1}(x)
\end{array}\right), \\
& \mathcal{B}(x)=\left(\begin{array}{cc}
B_{+}^{11}(x) & 0 \\
0 & -B_{-}^{11}(x)
\end{array}\right), \\
& F(x)=\left(\begin{array}{c}
f_{+}(x) \\
-f_{-}(x)
\end{array}\right), \\
& \Gamma W=V_{+}-V_{-} .
\end{aligned}
$$

In what follows, we shall keep track of variables $W$ as $\left(W_{1}^{+}, W_{2}^{+}, W_{1}^{-}, W_{2}^{-}\right) \in \mathbb{C}^{n-r} \times \mathbb{C}^{r} \times$ $\mathbb{C}^{n-r} \times \mathbb{C}^{r}$ in the obvious way corresponding to matrix blocks as above. Notice also that $\mathcal{B}$ is degenerate in $W_{1}^{ \pm}$-blocks.

Let $\mathcal{E}_{-}(0)$ be the space of boundary values of decaying solutions of (B.4) when $F=0$. Then, we have

$$
\operatorname{dim} \mathcal{E}_{-}(0)=i
$$

where $i$ is defined at the beginning of Section 1.2. On the other hand, ker $\Gamma$ has dimension $n$. Thus, Assumption (D) then implies that $\operatorname{ker} \Gamma$ and $\mathcal{E}_{-}(0)$ have an $l=i-n$ dimensional intersection spanned by

$$
\left(\phi_{1}(0), \phi_{1}(0)\right), \cdots,\left(\phi_{l}(0), \phi_{l}(0)\right)
$$

where functions $\phi_{i}(0)$ are defined as in the paragraph just below (A.3).

We define an augmented boundary condition $\tilde{\Gamma}$ with property that

$$
\mathbb{C}^{2 n}=\operatorname{ker} \tilde{\Gamma} \oplus \mathcal{E}_{-}(0) .
$$

Since $\phi_{j}$ form a basis of the tangential space of the smooth manifold $\left\{\bar{U}^{\delta}(\cdot)\right\}$ defined as in (H3), without loss of generality, we assume that the $j^{\text {th }}$ component of $\phi_{j}$ is not zero. Thus, let us define

$$
\tilde{\Gamma} W=\left(W_{1}, \cdots, W_{l}, V_{+}-V_{-}\right)
$$


where $W_{1}, \cdots, W_{l}$ are the first $l$ components of $W \in \mathbb{C}^{2 n}$.

Now we consider the system

$$
\begin{aligned}
& \mathcal{B} W_{x}-\mathcal{A} W=F \\
& \tilde{\Gamma} W=0 \quad \text { on } \quad\{x=0\} .
\end{aligned}
$$

Since any solution of $(\mathrm{B.8})$ is also a solution of (B.4), we only need to give an estimate for solutions of (B.8). By using the MZ conjugation [MeZ1], there is a uniformly bounded transformation $\mathcal{C}$ such that by setting $W=\mathcal{C} Z$, (B.8) gives

$$
\begin{aligned}
& \mathcal{B}(\infty) Z_{x}-\mathcal{A}(\infty) Z=\bar{F} \\
& \bar{\Gamma} Z=0 \quad \text { on } \quad\{x=0\}
\end{aligned}
$$

where $\bar{\Gamma}=\tilde{\Gamma} \mathcal{C}$. Now let us define new variable $Y$ as

$$
Y:=\mathcal{Q} Z, \quad \text { with } \quad \mathcal{Q}:=\left(\begin{array}{cc}
\left(\begin{array}{cc}
I_{n-r} & 0 \\
b_{1}^{11} & b_{2}^{11}
\end{array}\right) & 0 \\
& 0
\end{array} \quad\left(\begin{array}{cc}
I_{n-1} & 0 \\
-b_{1}^{11} & -b_{2}^{11}
\end{array}\right)\right)
$$

Then $Y$ solves

$$
\begin{aligned}
& \tilde{Y}_{x}-\mathcal{A}(\infty) \mathcal{Q}^{-1} Y=\hat{F} \\
& Y=\left(Y_{1}^{+}, Y_{2}^{+}, Y_{1}^{-}, Y_{2}^{-}\right) \\
& \tilde{Y}=\left(0, Y_{2}^{+}, 0, Y_{2}^{-}\right) \\
& \hat{\Gamma} Y=0 \quad \text { on } \quad\{x=0\}
\end{aligned}
$$

where $\hat{\Gamma}=\tilde{\Gamma} \mathcal{C} \mathcal{Q}^{-1}$ and $\hat{F}=\bar{F} \mathcal{Q}^{-1}$.

Now by view of (B.5), (1.3), and (H2), eigenvalues $\mu_{j}$ of each block of $\mathcal{A}(\infty) \mathcal{Q}^{-1}$ are distinct and nonzero. Thus, by performing a further transformation if necessary, we could assume that $\mathcal{A}(\infty) \mathcal{Q}^{-1}$ is diagonal. In these diagonalized coordinates, the system (B.10) consists of $2 n$ "uncoupled" equations:

$$
\begin{aligned}
-\mu_{j \pm} Y_{1 \pm} & =\hat{F}_{1 \pm} \\
\left(Y_{2 \pm}\right)_{x}-\mu_{j \pm} Y_{2 \pm} & =\hat{F}_{2 \pm}
\end{aligned}
$$

where note that $Y_{i \pm}$ are the projections of $Y_{i}$ on the growing (resp. decaying) eigenspaces of $\mathcal{A}$ associated to eigenvalues $\mu_{j \pm}$. In particular, $\pm \mu_{j \pm}>0$.

From equations for $Y_{1}$, it is clear that

$$
\left|Y_{1}\right|_{L^{p}} \lesssim\left|\tilde{F}_{1}\right|_{L^{p}} \quad \forall p \geq 1
$$

Meanwhile, $Y_{2 \pm}$ satisfies

$$
\begin{aligned}
& Y_{2+}(x)=\int_{x}^{\infty} e^{\mu_{j+}(x-y)} \hat{F}_{2+}(y) d y \\
& Y_{2-}(x)=e^{\mu_{j-} x} Y_{2-}(0)+\int_{0}^{x} e^{\mu_{j-}(x-y)} \hat{F}_{2-}(y) d y .
\end{aligned}
$$


Thus, this yields

$$
\begin{aligned}
\left|Y_{2+}\right|_{L^{p}} & \lesssim\left|\hat{F}_{2+}\right|_{L^{p}}, \\
\left|Y_{2-}\right|_{L^{p}} & \lesssim\left|\hat{F}_{2-}\right|_{L^{p}}+\left|Y_{2-}(0)\right|, \quad \forall p \geq 1 .
\end{aligned}
$$

Now since $Y_{2-}(0) \in \mathcal{E}_{-}(0)$, by view of $(\overline{\mathrm{B} .6})$ as bounded projections and the fact that all our transformations and their inverses are bounded, we must have

$$
\left|Y_{2-}(0)\right| \lesssim\left|\hat{\Gamma} Y_{-}(0)\right| \lesssim|\hat{\Gamma} Y(0)|+\left|Y_{+}(0)\right| \lesssim\left|Y_{+}\right|_{L^{\infty}}
$$

Altogether, we obtain

$$
|Y|_{L^{p}} \lesssim|\tilde{F}|_{L^{1}}+|\tilde{F}|_{L^{\infty}}, \quad \forall p \geq 1
$$

which proves the first bound in (B.2). Estimates for derivatives are then immediate by differentiating the equations of $Y_{1}$ in $(\underline{B .10})$ and by solving equations (B.10) for $Y_{2 x}$ in terms of $Y$ and $\hat{F}$. Thus, we have proved the lemma as claimed.

\section{Appendix C. Independence of the pointwise Green bounds}

In this section we comment on independence of the pointwise Green function estimates. The high-frequency estimate (2.4) can be derived entirely from auxiliary nonlinear energy estimates as done in [Z4]; see also Proposition 3.6, [NZ2], for a great simplification. Whereas, the independency for the low-frequency estimate (2.2) can be seen by first proving the following slightly-weaker version of Proposition 2.2, independent of the pointwise bounds (2.27),(2.28). A similar version can be done for Proposition 4.1.

Proposition C.1 (Low-frequency bounds). Under the hypotheses of Theorem 1.8, for $\lambda \in$ $\Gamma^{\tilde{\xi}}$ and $\rho:=|(\tilde{\xi}, \lambda)|, \theta_{1}$ sufficiently small, there holds the resolvent bound

$$
\left|\left(L_{\tilde{\xi}}-\lambda\right)^{-1} \partial_{x_{1}}^{\beta} f\right|_{L^{p}\left(x_{1}\right)} \leq C \rho^{-3 / 2+(1-\alpha) \beta}\left(|f|_{L^{1}\left(x_{1}\right)}+\left|\partial_{x_{1}} f\right|_{L^{1}\left(x_{1}\right)}\right),
$$

for all $2 \leq p \leq \infty, \beta=0,1$, and $\alpha$ defined as in (2.3).

Proof. Certainly by Proposition 2.2 , we only need to prove the bound in the case $\beta=1$. In the case of undercompressive shocks, the bound is clear by applying (C.1) with $\beta=0, \alpha=1$ and $f$ replaced by $\partial_{x_{1}} f$ :

$$
\left|\left(L_{\tilde{\xi}}-\lambda\right)^{-1} \partial_{x_{1}} f\right|_{L^{p}\left(x_{1}\right)} \leq C \rho^{-3 / 2}\left|\partial_{x_{1}} f\right|_{L^{1}\left(x_{1}\right)} .
$$

Now for the case of Lax or overcompressive shocks, we use the Kreiss-Kreiss trick as in the proof of Proposition [2.2, that is, write $U=V+U_{1}$ where $V$ solves the auxiliary problem (B.1). From the estimate (B.2) of $V$ and the inequality: $|f|_{L^{\infty}} \leq\left|\partial_{x_{1}} f\right|_{L^{1}\left(x_{1}\right)}$ (with $f(+\infty)=0)$, we have

$$
|V|_{L^{p}}+\left|V_{x}\right|_{L^{1}} \leq C\left(|f|_{L^{1}\left(x_{1}\right)}+\left|\partial_{x_{1}} f\right|_{L^{1}\left(x_{1}\right)}\right), \quad \forall p \geq 1 .
$$

Thus, replacing (2.29) by this inequality and following the proof of Proposition 2.2, we obtain the bound (C.1). 
Proof of Theorem 1.8, provided (C.1). The resolvent estimate (C.1) is only weaker than (2.18) by a stronger norm on $f$. We thus can certainly follow the proof in Section 2.4. yielding a low-frequency estimate like (2.2), but again weaker by a stronger norm on $f$, namely, $|f|_{L^{1}(x)}+\left|\partial_{x_{1}} f\right|_{L^{1}(x)}$. With this slightly weaker estimate for $\mathcal{S}_{1}$, we can follow word by word the proof of the theorem in Section 3 , noting that the higher derivatives of $f$ (then of $U$ ) can then be estimated by the energy estimate (3.6). Thus, we obtain the theorem without requiring any further regularity on the structures of the system.

\section{REFERENCES}

[GMWZ1] O. Guès, G. Métivier, M. Williams, and K. Zumbrun. Multidimensional viscous shocks I: degenerate symmetrizers and long time stability, J. Amer. Math. Soc. 18 (2005), no. 1, 61-120.

[GMWZ5] O. Guès, G. Métivier, M. Williams, and K. Zumbrun. Existence and stability of noncharacteristic hyperbolic-parabolic boundary-layers. Preprint, 2008.

[GMWZ6] O. Guès, G. Métivier, M. Williams, and K. Zumbrun. Viscous boundary value problems for symmetric systems with variable multiplicities, J. Differential Equations 244 (2008) 309387.

[HoZ1] D. Hoff and K. Zumbrun, Multi-dimensional diffusion waves for the Navier-Stokes equations of compressible flow, Indiana Univ. Math. J. 44 (1995), no. 2, 603-676.

[HoZ2] D. Hoff and K. Zumbrun, Pointwise decay estimates for multidimensional Navier-Stokes diffusion waves, Z. Angew. Math. Phys. 48 (1997), no. 4, 597-614.

[HLyZ1] Humpherys, J., Lyng, G., and Zumbrun, K., Spectral stability of ideal-gas shock layers, Preprint (2007).

[HLyZ2] Humpherys, J., Lyng, G., and Zumbrun, K., Multidimensional spectral stability of large-amplitude Navier-Stokes shocks, in preparation.

[KK] Kreiss, G. and Kreiss, H.-O., Stability of systems of viscous conservation laws, Comm. Pure Appl. Math., 50, 1998, 1397-1424.

[KZ] B. Kwon and K. Zumbrun, Asymptotic Behavior of Multidimensional scalar Relaxation Shocks, Preprint, 2008

[MaZ3] C. Mascia and K. Zumbrun. Pointwise Green function bounds for shock profiles of systems with real viscosity. Arch. Ration. Mech. Anal., 169(3):177-263, 2003.

[MaZ4] C. Mascia and K. Zumbrun. Stability of large-amplitude viscous shock profiles of hyperbolicparabolic systems. Arch. Ration. Mech. Anal., 172(1):93-131, 2004.

[MeZ1] Métivier, G. and Zumbrun, K., Large viscous boundary layers for noncharacteristic nonlinear hyperbolic problems, Memoirs AMS, 826 (2005).

[MeZ3] Métivier, G. and Zumbrun, K., Hyperbolic boundary value problems for symmetric systems with variable multiplicities, J. Diff. Eqns., 211, (2005), 61-134.

[N2] T. Nguyen, On asymptotic stability of noncharacteristic viscous boundary layers, Preprint, 2009.

[NZ2] T. Nguyen and K. Zumbrun, Long-time stability of multi-dimensional noncharacteristic viscous boundary layers, Preprint, 2008

[Z2] K. Zumbrun. Multidimensional stability of planar viscous shock waves. In Advances in the theory of shock waves, volume 47 of Progr. Nonlinear Differential Equations Appl., pages 307-516. Birkhäuser Boston, Boston, MA, 2001.

[Z3] K. Zumbrun. Stability of large-amplitude shock waves of compressible Navier-Stokes equations. In Handbook of mathematical fluid dynamics. Vol. III, pages 311-533. North-Holland, Amsterdam, 2004. With an appendix by Helge Kristian Jenssen and Gregory Lyng.

[Z4] K. Zumbrun. Planar stability criteria for viscous shock waves of systems with real viscosity. In Hyperbolic systems of balance laws, volume 1911 of Lecture Notes in Math., pages 229-326. Springer, Berlin, 2007.

Department of Mathematics, Indiana University, Bloomington, IN 47405

E-mail address: nguyentt@indiana.edu 